\title{
1 Natural language syntax complies with the free-energy principle
}

3 Elliot Murphy ${ }^{1,2}$, Emma Holmes ${ }^{3,4}$, Karl Friston ${ }^{4}$

4 1. Vivian L. Smith Department of Neurosurgery, McGovern Medical School, 5 University of Texas Health Science Center, Houston, Texas, 77030, USA

2. Texas Institute for Restorative Neurotechnologies, University of Texas Health 7 Science Center, Houston, Texas, 77030, USA

3. Department of Speech Hearing and Phonetic Sciences, University College London, London, WC1N 1PF, UK

4. The Wellcome Centre for Human Neuroimaging, UCL Queen Square Institute of Neurology, London, WC1N 3AR, UK

13 Correspondence concerning this article should be addressed to Elliot Murphy 14 (elliot.murphy@uth.tmc.edu).

15 Word count: 13,443

16 Figure count: 1 


\section{Abstract:}

18 Natural language syntax yields an unbounded array of hierarchically structured expressions. We claim that these are used in the service of active inference in accord with the free-energy principle (FEP). While conceptual advances alongside modelling and simulation work have attempted to connect speech segmentation and linguistic communication with the FEP, we extend this program to the underlying computations responsible for generating syntactic objects. We argue that recently proposed principles of economy in language design—such as "minimal search" criteria from theoretical syntax-adhere to the FEP. This affords a greater degree of explanatory power to the FEP—with respect to higher language functions-and offers linguistics a

27 grounding in first principles with respect to computability. We show how both treegeometric depth and a Kolmogorov complexity estimate (recruiting a Lempel-Ziv compression algorithm) can be used to accurately predict legal operations on syntactic workspaces, directly in line with formulations of variational free energy minimization. This is used to motivate a general principle of language design that we term TuringChomsky Compression (TCC). We use TCC to align concerns of linguists with the normative account of self-organization furnished by the FEP, by marshalling evidence

34 from theoretical linguistics and psycholinguistics to ground core principles of efficient syntactic computation within active inference.

Keywords: Free-energy principle; active inference; language; Kolmogorov

37 complexity; Lempel-Ziv; compression 
Implementational models of language must be plausible from the perspective of neuroanatomy (Embick \& Poeppel 2015), but they must also be plausible from the

40

perspective of how biophysical systems behave. We will argue that the structuring influence of the free-energy principle (FEP) can be detected in language, not only via narrative (Bouizegarene et al. 2020), interpersonal dialogue (Friston et al. 2020), cooperative/intentional communication (Vasil et al. 2020) and speech segmentation (Friston et al. 2021), but also at the more fundamental level of what linguists consider to be basic phrase-level computations (Berwick \& Stabler 2019, Chomsky 1949, 1951, 1956, 1959, 2021a, 2021b, 2021c).

Natural language syntax yields an unbounded array of hierarchically structured expressions. We argue that many historical insights into syntax are consistent with the FEP_-providing a novel perspective under which the principles governing syntax are not limited to language, but rather reflect domain-general processes. This is consistent with a strain within theoretical linguistics that explores how syntactic computation may adhere to "general principles that may well fall within extra-biological natural law, particularly considerations of minimal computation" (Chomsky 2011: 263), such that certain linguistic theories might be engaging with general properties of organic systems (Chomsky 2004, 2014). Here, we consider the idea that many aspects of natural language syntax may be special cases of a variational principle of least freeenergy. To this end, we examine whether a complexity measure relevant to formulations of free-energy—namely, Kolmogorov complexity (Hutter 2006, MacKay 1995, Wallace \& Dowe 1999)—relates to legal syntactic processes.

While the FEP has a substantial explanatory scope, across a large range of cognitive systems, it can also be seen as a method or principle of least action for multi- 
62 disciplinary research (Ramstead et al. 2021), in much the same way that the notion of

63 economy is typically entertained in linguistics as a programmatic notion (Chomsky 64 1995). The FEP itself has been argued to be more of a conceptual-mathematical 65 model for self-organizing systems (for some, it is a "generic" model; Barandiaran \& Chemero 2009), or a guiding framework (Colombo \& Wright 2021). Thus, when we argue that natural language syntax "complies" with the FEP, this is not to imply that the FEP necessarily bears any specific, direct predictions for linguistic behaviour. Rather, it motivates the construction of conceptual arguments for how some property of organic systems might be seen as realizing the FEP.

We begin by summarising the FEP, and describe how syntactic principles are consistent with it. We consider how the FEP is a variational principle of "least action", such as those that describe systems with conserved quantities (Coopersmith 2017). We then review key observations from linguistics that speak to the structuring influence of computational efficiency, involving "least effort" and "minimal search" restrictions (Bošković \& Lasnik 2007, Gallego \& Martin 2018, Larson 2015), viewing 77 language as a product of an individual's mind/brain, following the standard 'I-language' 78 (Chomsky 1986, 2000) perspective in generative linguistics (i.e., 'internal', 'individual', 79 'intensional'). After modeling the complexity of postulated minimal search procedures-versus their ungrammatical alternatives across a small but representative number of exemplar cases-we propose a unifying principle for how the goals of the FEP might be realised during the derivation of syntactic structures, 83 which we term Turing-Chomsky Compression. We conclude by highlighting directions 84 for future work. 


\section{Active Inference and the Free-Energy Principle}

87 Before we evaluate any work pertaining to linguistic behaviour, this section introduces key elements of the FEP that motivate its application to language.

89

90

\subsection{The Free-Energy Principle}

91 The FEP has a long history in theoretical neuroscience (see Friston 2010 for a review).

92 It states that any adaptive change in the brain will minimise free-energy, either over 93 evolutionary time or immediate, perceptual time (Ramstead et al. 2018). Free-energy 94 is an information-theoretic quantity and is a function of sensory data and brain states: 95 in brief, it is the upper bound on the 'surprise'-or surprisal (Tribus 1961) —of sensory data, given predictions that are based on an internal model of how those data were 97 generated. The difference between free-energy and surprise is the difference 98 (specified by the Kullback-Leibler divergence) between probabilistic representations encoded by the brain and the true conditional distribution of the causes of sensory

100 input. This is evident in the following equation, which specifies variational free energy

$101(F)$ as the negative log probability of observations $(\tilde{O})$ under a generative model (i.e., 102 'surprise') plus the Kullback-Leibler divergence ( $\left.D_{K L}\right)$ between the approximate 103 posterior distribution and the true posterior distribution (where $Q$ indicates posterior 104 beliefs, $\tilde{S}$ indicates the states in the generative model, and $P$ indicates the probability 105 under the internal model):

$$
F=-\ln P(\tilde{o})+D_{K L}[Q(\tilde{s}) \| P(\tilde{s} \mid \tilde{o})]
$$


107 Unlike surprise itself, variational free energy can be evaluated (for a detailed 108 explanation, see Friston, FitzGerald et al. 2017). Under simplifying assumptions, free109 energy can be considered as the amount of prediction error; for a mathematical 110 comparison, see Friston, Parr et al. (2017). Minimising free energy minimises surprise, and is equivalent to maximising the evidence for the internal model of how sensory data were generated. By minimising free-energy, the brain is essentially performing approximate Bayesian inference. By reformulating variational free energy-in a way

114 that is mathematically equivalent; see Penny et al. (2004), Winn \& Bishop (2005)—we

115 see that free-energy can be considered as a trade-off between accuracy and complexity, whereby the best internal model is the one that accurately describes the data in the simplest manner (where $E_{Q}$ indicates the expected value, and the other variables are the same as those defined above):

$$
\begin{aligned}
F & =E_{Q}[\ln Q(\tilde{s})-\ln P(\tilde{o} \mid \tilde{s})-\ln P(\tilde{s})] \\
& =E_{Q}[\ln Q(\tilde{s})-\ln P(\tilde{s} \mid \tilde{o})-\ln P(\tilde{o})] \\
& =\underbrace{D_{K L}[Q(\tilde{s}) \| P(\tilde{s} \mid \tilde{o})]}_{\text {relative entropy }}-\underbrace{\ln P(\tilde{o})}_{\text {log evidence }} \\
& =\underbrace{D_{K L}[Q(\tilde{s}) \| P(\tilde{s})]}_{\text {complexity }}-\underbrace{E_{Q}[\ln P(\tilde{o} \mid \tilde{s})]}_{\text {accuracy }}
\end{aligned}
$$

120 Because the relative entropy can never be less than zero, the variational free energy

121 provides an upper bound on negative log evidence: equivalently, the negative free 122 energy provides a lower bound on log evidence; known as an evidence lower bound 123 (ELBO) in machine learning (Winn \& Bishop 2005). The final equality shows a complementary decomposition of variational free energy into accuracy and complexity. In effect, it reflects the degree of belief updating afforded by some new sensory data; in other words, how much some new evidence causes one to "change 
127 one's mind". A good generative model—with the right kind of priors-will minimise the 128 need for extensive belief updating and thereby minimise complexity.

The complexity part of variational free energy will become important later, when

130 we will be evaluating the complexity of syntactic processes using a measure derived both in spirit and mathematical heritage from the foundations of the FEP. To present some initial details about this, consider how complexity also appears in treatments of

133 universal computation (Hutter 2006) and in the guise of minimum message or 134 description lengths (Wallace \& Dowe 1999). Indeed, in machine learning, variational free energy minimisation has been cast as minimising complexity_or maximising efficiency in this setting (MacKay 1995). One sees that same theme emerge in predictive coding-and related-formulations of free energy minimisation, where the underlying theme is to compress representations (Schmidhuber 2010), thereby maximising their efficiency (Barlow 1961, Linsker 1990, Rao \& Ballard 1999). We will return to these topics below when we begin to formalise features of natural language syntax.

Lastly, the FEP can also be considered from the perspective of a Markov 143 blanket (see Kirchhoff et al. 2018, Palacios et al. 2020, Parr et al. 2020 for detailed explanation), which instantiates a statistical boundary between internal states and external states. In other words, internal (e.g., in the brain) and external (e.g., in the external world) states are conditionally independent: they can only influence one

147 another through blanket states. The blanket states can be partitioned into sensory 148 states and active states. External states affect internal states only through sensory 149 states, while internal states affect external states only through active states. The 150 implicit circular causality is formally identical to the perception-action cycle (Fuster 
2004). Under previous accounts (Friston, FitzGerald et al. 2017), the brain can

152 minimise free-energy either through perception or action. The former refers to

153 optimising (i.e., using approximate Bayesian inference to invert) its probabilistic

154 generative model that specifies how hidden states cause sensory data; in other words,

155 inferring the cause of sensory consequences by minimising variational free energy.

156 The latter refers to initiating actions to sample data that are predicted by its model-

157 which we turn to next.

158

\section{2. $\quad$ Active Inference}

160 The enactive component of active inference rests on the assumption that action is

161 biased to realize preferred outcomes. Beliefs about which actions are best to pursue

162 rely on predictions about future outcomes, and the probability of pursuing any 163 particular outcome is given by the expected free energy of that action. Expected free 164 energy $(G)$ can be expressed as the combination of extrinsic and epistemic value 165 (Friston, Parr et al. 2017), where $\pi$ is a series of actions (i.e., the policy) being pursued, $166 \tau$ is the current time point, and the other variables are the same as those defined 167 above:

$$
\begin{aligned}
G(\pi) & =\sum_{\tau} G(\pi, \tau) \\
G(\pi, \tau) & =E_{Q}\left[\ln Q\left(s_{\tau} \mid \pi\right)-\ln Q\left(s_{\tau} \mid o_{\tau}, \pi\right)-\ln P\left(o_{\tau}\right)\right] \\
& =\underbrace{E_{Q}\left[\ln Q\left(s_{\tau} \mid \pi\right)-\ln Q\left(s_{\tau} \mid o_{\tau}, \pi\right)\right]}_{\text {(negative) mutual information }}-\underbrace{E_{Q}\left[\ln P\left(o_{\tau}\right)\right]}_{\text {expected log evidence }} \\
& =\underbrace{E_{Q}\left[\ln Q\left(o_{\tau} \mid \pi\right)-\ln Q\left(o_{\tau} \mid s_{\tau}, \pi\right)\right]}_{\text {(negative) epistemic value }}-\underbrace{E_{Q}\left[\ln P\left(o_{\tau}\right)\right]}_{\text {extrinsic value }}
\end{aligned}
$$


169 Extrinsic value is the expected evidence for the internal model under a particular 170 course of action, whereas epistemic value is the expected information gain: in other 171 words, the extent a series of actions reduces uncertainty.

Notice that the expected versions of relative entropy and log evidence in the

173 free energy (Eq. 2) now become intrinsic and extrinsic value respectively (Eq. 3). As

174 such, selecting an action to minimise expected free energy reduces expected surprise

175 (i.e., uncertainty) in virtue of maximising the information gain while-at the same

176 time-maximising the expected log evidence; namely, actively self-evidencing (Hohwy

$1772016,2020)$. When applied to a variety of topics in cognitive neuroscience, active

178 inference has been shown to predict human behaviour and neuronal responses; e.g.,

179 Brown et al. (2013), Friston, Lin et al. (2017), Friston, Rosch et al. (2017), Parr and 180 Friston (2017, 2019), Smith et al. (2019).

As will soon become clear, we will be using these observations concerning complexity to motivate a specific application of these ideas to natural language syntax, utilizing a measurement of algorithmic complexity that shares a mathematical heritage

184 with the FEP, as outlined here.

\subsection{Belief Updating}

187 Belief updating refers to a process by which free energy is minimised. By specifying a process theory that explains neuronal responses during perception and action, neuronal dynamics have previously been cast as a gradient flow on free energy

190 (known as variational free energy in physics, introduced in Feynman 1972; see Hinton

191 \& Zemel 1994); we refer the reader to Friston, FitzGerald et al. (2017) for a treatment 
192 of neuronal message passing and belief propagation. That is to say, any neural

193 process can be formulated as a minimisation of the same quantity used in approximate

194 Bayesian inference (Andrews 2021, Hohwy 2016). We provide an example of the 195 computational architecture implied by this formulation of belief updating in the brain

196 (Fig. 1). This illustrative example is based upon a discrete state space generative

197 model, where the equations describe the solutions to Bayesian belief updating of 198 expectations pertaining to hidden states, policies, policy precision and parameters.

In short, the brain seeks to minimise free energy, which is mathematically equivalent to maximising model evidence. This view of neuronal responses can be

202 path integral of free energy.

\section{Functional anatomy and message passing}

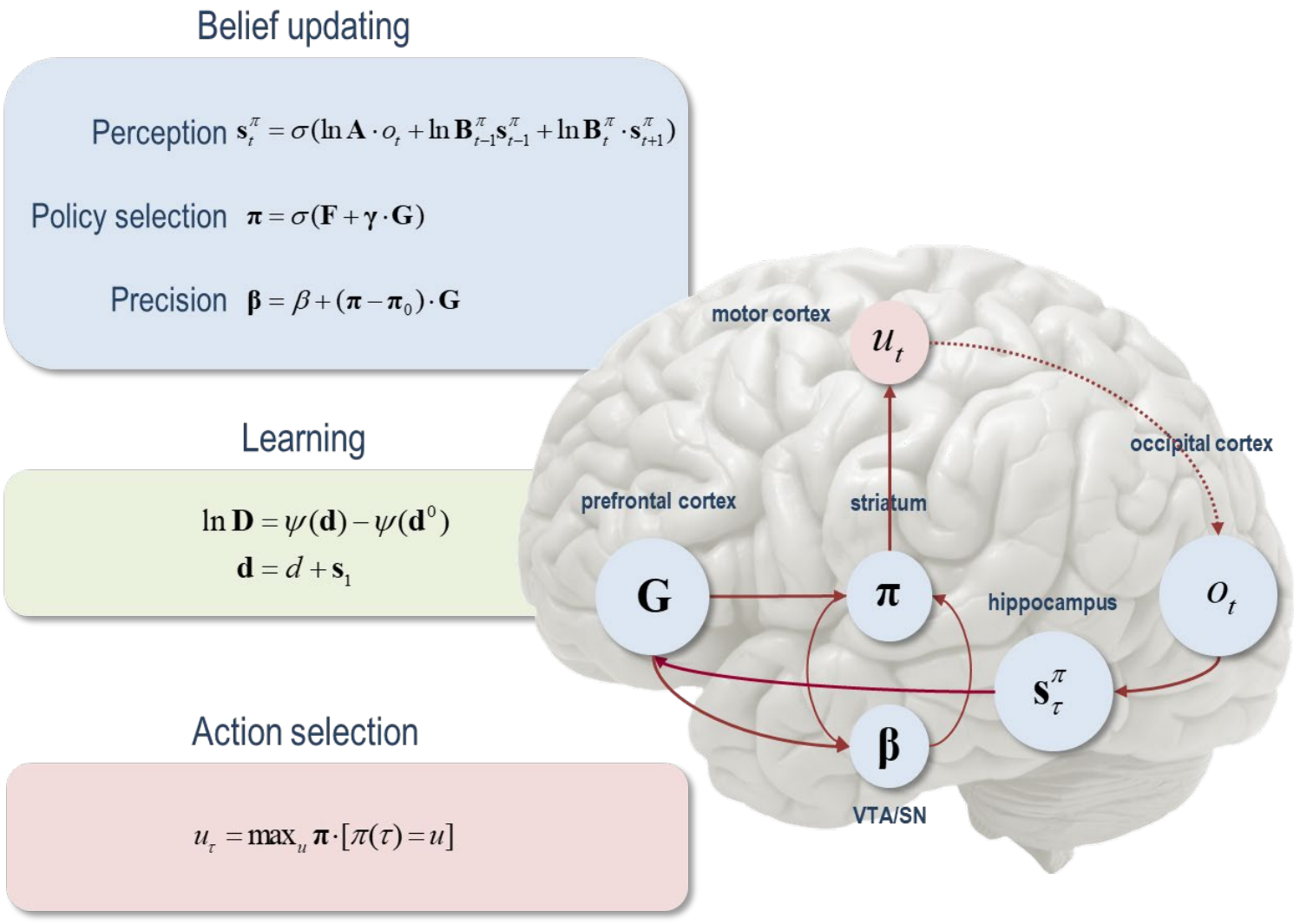


Figure 1: Schematic overview of belief updates for active inference under discrete

Markovian models. The left panel lists the belief updating equations, associating various updates with action, perception, policy selection, precision and learning. The left panel assigns the variables (sufficient statistics or expectations) that are updated to various brain areas. This attribution serves to illustrate a rough functional anatomy-implied by the form of the belief updates. In this simplified scheme, we have assigned observed outcomes to visual representations in the occipital cortex and updates to hidden states to the hippocampal formation. The evaluation of policies, in terms of their (expected) free energy, has been placed in the ventral prefrontal cortex. Expectations about policies per se and the precision of these beliefs have been attributed to striatal and ventral tegmental areas to indicate a putative role for dopamine in encoding precision. Finally, beliefs about policies are used to create Bayesian model averages over future states, which are fulfilled by action. The red arrows denote message passing. In brief, the parameters of the generative model correspond to matrices or arrays encoding the likelihood

A, prior state transitions

$\mathbf{B}$, and initial hidden states

D. $F$ corresponds to the free energy of each policy and $\mathbf{G}$ corresponds to the expected free energy, which is weighted by a precision or softmax parameter $\gamma$ that is usually attributed to dopaminergic neurotransmission. See Friston, FitzGerald et al. (2017) for further explanation

221 of the equations and variables.

\subsection{Previous Applications}

224 Applying active inference to language relies on finding the right sort of generative

225 model, and many different structures and forms of generative model are possible. Most

226 relevant to the current application, deep temporal models accommodate a nesting of 227 states that unfold at different temporal scales. Since language output is inherently temporal, this leads to the question of how to map hierarchical structures onto serial outputs (Epstein et al. 1998), and models that are deep in time allow us to deconstruct associated nested structures. 
233 behavioural and neurophysiological correlates of communication arise under 234 variational message passing (Friston et al. 2020). The model incorporates various 235 levels that operate at different temporal scales. At the lowest level, it specifies 236 mappings among syntactic units that, when combined with particular semantic beliefs, 237 predict individual words. At a higher level (i.e., longer temporal scales), the model 238 contains beliefs about the context it is in, which specifies the syntactic structure at the 239 level below. This model is congruent with core assumptions from linguistics concerning 240 the generative nature of language. Specifically, elementary syntactic units provide 241 belief structures that are used to reduce uncertainty about the world, through rapid 242 and reflexive categorization of events, objects and their relations. Then, sentential 243 representations can be thought of as structures designed (partially) to consolidate and 244 appropriately frame experiences, and to contextualise and anticipate future 245 experiences. The range of parseable syntactic structures available to comprehenders 246 provides alternate hypotheses that afford parsimonious explanations for sensory data 247 and, as such, preclude overfitting (Sengupta \& Friston 2018). If the complexities of 248 linguistic stimuli can be efficiently mapped to a small series of regular syntactic 249 formats, this contributes to the brain's goal of restricting itself to a limited number of 250 states. Intuitively, knowing that you can only have said this or that greatly simplifies 251 the inference problem, rendering exchanges between like-minded artefacts more 252 efficient and economic. Before moving forward, we stress here that we will be working within the 254 framework of theoretical linguistics (which deals with derivational stages of word-by255 word, element-wise operations that underlie sentences), and not a framework such as 256 corpus linguistics (which deals with the output of the generative/derivational process). 
257 Questions we raise here therefore cannot be addressed by consulting large corpora, 258 but instead require investigation of incremental computational steps that ultimately 259 appear to be responsible for the complex forms of human language behavior studied 260 by sociolinguistics, corpus linguistics, and historical linguistics. Relatedly, embracing 261 the traditional distinction between competence and performance, our focus will be on 262 the former (the mental formatting and generation of linguistic structure), and not on the 263 range of complex cognitive processes that enter into the use of language in a specific 264 context. The FEP has many implications for the study of language, and we focus here 265 on a very narrow, specific topic of derivational syntactic complexity (language 266 knowledge, as opposed to language use). Whether or not free energy is minimized across corpora, or across paragraphs from clippings of newspaper columns, or across the world's languages, is an open topic-but here we will focus on the computational system of human language.

\section{Computational Principles and Syntactic Hierarchies}

\subsection{A System of Discrete Infinity}

273 How can the FEP contribute to our understanding of syntactic computation? Most

274 immediately, it provides fundamental constraints on the physical realisation of a 275 computational system. Consider first the three principles in classical recursive function 276 theory which allow functions to compose (Kleene 1952): substitution; primitive 277 recursion; and minimisation. These are all designed in a way that one might think of 278 as computationally efficient: they reuse the output of earlier computations. Substitution 279 replaces the argument of a function with another function (possibly the original one); 
280 primitive recursion defines a new function on the basis of a recursive call to itself, 281 bottoming out in a previously defined function (Lobina 2017); minimisation (also 282 termed 'bounded search') produces the output of a function with the smallest number 283 of steps (see also Piantadosi 2021, for whom human thought is essentially like Church encoding). More broadly, we note that free energy minimization-by constructionentails Bayesian inference, which in turn is a computational process, and so the FEP entails computationalism (Korbak 2021) and at least a type of (basic) computational 287 architecture for language we assume here (but see Kirchhoff \& Robertson 2018). 288 Examining some core principles of recursion, natural language clearly exhibits minimisation, while binary branching of structures (Radford 2016) limits redundant computation, reducing the range of possible computations. Even limitations on shortterm memory have been hypothesized to contribute to the efficiency of memory search (MacGregor 1987). sets via the operation MERGE, taking objects from the lexicon or objects already part of the workspace. For example, given the set $\{\mathrm{X}, \mathrm{Y}\}$, we can either select a new lexical object and MERGE it, to form $\{Z,\{X, Y\}\}$, or we can select an existing object and MERGE it to the same workspace, to form $\{X,\{X, Y\}\}^{1}$. MERGE serves a similar role to an elementary function, as in the theory of computability (e.g., the zero function, the

1 There are recent debates concerning whether merging new lexical objects is more computationally demanding (since it requires searching the lexicon) than merging lexical objects that are already in the workspace. This may motivate a Move-over-Merge bias (reversing the Merge-over-Move assumption from the early minimalism of the 1990s), however we leave this issue to one side. 
299 identity function), in that it is meant to be non-decomposable. Putting many subsidiary 300 technical details aside, these sets are then 'labeled' and given a syntactic identity, or 301 a 'head' (Frampton \& Gutmann 1999, Murphy 2015a, Murphy, Woolnough et al. 2022), 302 based on which element is most structurally prominent and easiest to search for (i.e., $303 Z$ in the structure $\{Z,\{X, Y\}\})^{2}$. For example, 'the old man' is a Determiner Phrase (DP), 304 and has the distributional properties of a DP, since 'the' is equivalent to $Z$, and 'old 305 man' equivalent to $\{X, Y\}$. Labeling takes place when conceptual systems access the 306 structures generated by syntax. This occurs at distinct derivational punctuations based 307 on the configuration and subcategorization demands of the lexical items involved (e.g., 308 in many instances subjects seem to be featurally richer than objects, and provide the 309 relevant feature for the label; Longobardi 2008). For example, in the case of head310 complement structures this is done immediately after MERGE (Bošković 2016). 311 MERGE can also derive some set-theoretic properties of linguistic relations, such as 312 membership, dominate and term-of, as well as the derived relation of c-command 313 (=sister of) which is relevant for interpreting hierarchical relations between linguistic 314 elements (Haegeman 1994) ${ }^{3}$. These also appear to be the simplest possible formal 315 relations entertained, potentially indexing a feature of organic computation that 316 adheres closely to criteria of simplicity (Chomsky 2022).

2 There is increasing evidence that only elements in the workspace that have been labeled can be subject to movement (Bošković 2021).

${ }^{3}$ Note that there are also numerous cases in which principles previously seen as purely syntactic have been re-framed as, for example, phonological in nature (e.g., string adjacency; Bobaljik 2002; see also Richards 2016, Samuels 2011). 
318 the removal of superfluous degrees of freedom in order to describe a given physical

319 system at a different scale), with the core process of syntax being information 320 renormalization according to different timescales. For instance, MERGE can be

321 framed as a probability tensor implementing coarse-graining, akin to a probabilistic 322 context-free grammar (Gallego \& Orús 2022). The model proposed in Gallego and 323 Orús (2022: 20) assumes that language is "the cheapest non-trivial computational 324 system", exhibiting a high degree of efficiency with respect to its MERGE-based coarse-graining.

Natural language syntax exhibits discrete units which lead to a discretenesscontinuity duality (the boundary between syntactic categories can be non-distinct) ${ }^{4}$.

328 Syntax is driven by closeness of computation (syntactic objects $\mathrm{X}$ and $\mathrm{Y}$ form a distinct 329 syntactic object, $\{X, Y\}$ ). Its objects are bounded (a fixed list, e.g., N, V, Adj, Adv, P, $330 \mathrm{C}, \mathrm{T}, n, v, \mathrm{Asp}, \mathrm{Cl}, \mathrm{Neg}, \mathrm{Q}$, Det) and their hierarchical ordering is based on a specific 331 functional sequence such as C-T-v-V (e.g., C is always higher than V; Starke 2004) 332 which imposes direct restrictions on combinatorics (Adger \& Svenonius 2011). These 333 objects can be combined in cycles (Frampton \& Gutmann 1999), which can be

4 In active inference, the use of discrete-as opposed to continuous-states in generative models is an enormously potent way of minimising complexity. For example, if it is sufficient to carve the world (i.e., the causes of my sensations) into a small number of hidden states, one can minimise the complexity of belief updating by not redundantly representing all the fine-grained structure within any one state. Similarly, factorisation plays a key role in structuring our hypotheses or expectations that provide the best explanation for sensations. Perhaps the clearest example here is the carving of a sensory world into what and where. This means one does not have to represent the location of every possible object in one's visual cortical hierarchy, just where an object is and what an object is-and then integrate these representations to explain visual input (Ungerleider \& Haxby 1994). 
extended to form non-local dependencies. As we will discuss, these properties are guided by principles of minimal search (an optimal tree-search procedure, informed by notions from computer science; Aycock 2020, Ke 2019, Roberts 2019) and least effort

337 (Larson 2015), fulfilling the imperatives of active inference to construct meaningful representations as efficiently as possible (Bouizegarene et al. 2020), contributing to surprise minimisation.

\subsection{Compositionality}

342 Recently, certain efficiency principles at the conceptual interface (where syntax

343 interfaces with general conceptualization) have been proposed (Pietroski 2018), such

344 that the 'instructions' that language provides for the brain to build specific meanings are interpreted with notable simplicity. Leaving more technical details aside, this is ultimately achieved (in Pietroski's model) through M-join (e.g., $F\left(\_\right)+G\left(\_\right) \rightarrow F^{\wedge} G\left(\_\right)$, which combines monadic concepts, like red + boat) and D-join (e.g., D(_,_) + M(_) $\rightarrow$ $348 \exists\left[\mathrm{D}\left({ }_{-},\right)^{\wedge} \mathrm{M}\left(\_\right)\right]$, which merges dyadic concepts with monadic concepts, deriving the 349 meaning of $X$ verb(ed) $Y$ ). Hence, natural language permits limited dyadicity as a very minimal departure from the most elementary monadic combinatorial system. Human language is marginally more expressive (in its conceptual interpretations) than a firstorder language (i.e., one set, and one embedding), but the interpretation system is the

353 least complex needed to express dyadicity and permit relations between sets. As with models of syntax invoking a simple process of binary set-formation to derive recursion, by restricting the number of computational procedures able to generate semantic structures, this model (and others like it; Heim \& Kratzer 1998) restricts in highly predictable ways the possible range of outputs. This model also assumes that there 
are 'fetchable' concepts that language can use for compositionality, and non-fetchable concepts. For instance, language seems to make considerable use of certain contentful concepts (e.g., evidentiality) but not others (e.g., colour, worry/sollicitativity) 361 (Adger 2019, Peterson 2016). to predicates of certain semantic types (Schein 1993, Pietroski 2005). Certain semantic rules of composition, in (1b), have been claimed to arise directly from more elementary syntactic computations (Pietroski 2018) which adhere to principles of efficient computation.

(1) a. Dutch shot Micah quickly.

In this connection, it has further been observed that language acts as an artificial context which helps "constrain what representations are recruited and what 371 impact they have on reasoning and inference" (Lupyan \& Clark 2015: 283). Words

372 themselves are "highly flexible (and metabolically cheap) sources of priors throughout 373 the neural hierarchy" (Ibid) (for discussion of simplicity in semantic computation, see 374 Al-Mutairi 2014, Bošković \& Messick 2017, Collins 2020, Gallego \& Martin 2018, 375 González Escribano 2005, Hauser et al. 2002, Hornstein \& Pietroski 2009). The notion 376 of truth is also deeply wedded to complex syntax (Hinzen 2016, Hinzen \& Sheehan 377 2013), and is a notion that enormously increases an agent's ability to generate 378 environmental inferences.

To briefly elaborate on the language/meaning relation, consider how universal 380 syntactic hierarchies such as Complementizer Phrases (CPs) containing Tense 
381 Phrases (TPs), and TPs containing Verb Phrases (VPs) (i.e., [CP[TP[VP]]]), can be

382 thought to emerge directly from extra-linguistic conceptual relations, or the "cognitive

383 proclivity to perceive experience in terms of events, situations, and propositions" 384 (Ramchand \& Svenonius 2014: 33). An event (VP) is a kind of timeless description 385 (e.g., 'run'), and when an event is merged with a deictic anchor the result is a situation 386 (TP), a time-anchored eventuality. Only when a situation is merged with a specific 387 discourse link does it become a proposition (CP), providing speaker perspectives (e.g., 388 'John will run').

\section{A Kolmogorov Complexity Estimate for Narrow Syntax}

\subsection{Economy}

392 The notion of simplicity has been a methodological value which has guided linguistic 393 inquiry for decades (Terzian \& Corbalan 2021). Chomsky (2021b: 13) notes that 394 measures of simplicity in linguistics have traditionally focused on symbol counting, 395 which transpired to be too crude a measure, and that "measuring simplicity is an essential task and is no simple matter". We aim in this section to elaborate a measure of syntactic complexity that connects directly to the principles that underwrite active 398 inference. We will not be concerned with typological, phonological or acquisitional 399 notions of complexity, which form the bulk of current complexity literature. Instead, we 400 are interested in underlying representational issues that pertain to syntax-semantics. 401 Even the most recent explorations of simplicity in language, such as the volume on simplicity in grammar learning in Katzir et al. (2021), focuses on modelling minimum 
description length in phonology and morphology, but not processes pertaining to the 404 derivation of syntactic objects.

A number of economy principles have been proposed in theoretical linguistics:

406 the No Tampering Condition (Chomsky 2008), Minimal Link Condition (Chomsky 407 1995), Minimal Yield (Chomsky 2021c), Extension Condition (Chomsky 1995), Last 408 Resort (Chomsky 1995), Relativised Minimality (Rizzi 1990, 2001), Inclusiveness 409 Condition (Chomsky 1995), Precedence Resolution Principle (Epstein et al. 1998), 410 Scope Economy (Fox 2000), Phase Impenetrability Condition (Chomsky 2004), Full 411 Interpretation (Freidin \& Lasnik 2011, Lohndal \& Uriagereka 2016), Global Economy 412 Condition (Sternefeld 1997), Feature Economy (van Gelderen 2011), Accord 413 Maximization Principle (Schütze 1997), Input Generalisation (Holmberg \& Roberts 414 2014), Maximise Minimal Means (Biberauer 2019a), Resource Restriction (Chomsky 415 et al. 2019), and Equal Embedding (Murphy \& Shim 2020) (for further discussion, see 416 Frampton \& Gutmann 1999, Fukui 1996, Titov 2020). Although economy principles have figured in models of phonology, morphology 418 and the lexicon (e.g., the Elsewhere condition, underspecification) for decades, it is 419 only relatively recently that theories of syntax have embraced economy not just as a 420 heuristic guiding research, but more concretely as a constitutive principle of language 421 design (Leivada \& Murphy 2021, Reuland 2011, Sundaresan 2020). These have been 422 framed within a linguistic context, often invoking domain-specific notions (Wilder et al. 423 1997), despite a core part of the intended project of modern theoretical linguistics 424 being to embed linguistic theory within principles general to cognition. Motivating 425 language-specific computational generalizations by direct reference to the FEP may 426 broaden the explanatory scope for the existence and prevalence of particular syntactic 
427 phenomena. Since linguists lack a general theory of computational efficiency for 428 language (e.g., Gallego \& Chomsky 2020: "To be sure, we do not have a general 429 theory of computational efficiency"), additional support with respect to grounding these 430 concerns within a well-motivated framework for general organic behaviour will likely 431 prove productive. There are many promising paths to take here: minimising energy 432 expenditure during language processing (Rahman \& Kaykobad 2005), shortening 433 description length (Schmidhuber 2015), reducing Kolmogorov complexity (Ming \& 434 Vitányi 2008, Wallace \& Dowe 1999), and the degree of requisite belief updating; 435 relatedly, we might consult the principles of minimum redundancy and maximum 436 efficiency in perception (Barlow 1961, 1974, Wipf \& Rao 2007). We will provide a 437 concrete exploration of one of these notions (Kolmogorov complexity) below.

A core fact about many natural language expressions is that they involve 439 arrangements of nested constituents that enter into relations and dependencies of 440 various kinds. How are syntactic operations compressed into determinate, 441 unambiguous instructions to conceptual systems, and are there any general laws of 442 organic design that can be inferred from the FEP that appear to constrain this process 443 (and which successfully predict which objects cannot be constructed)?

444 Consider how under the No Tampering Condition the merging of two syntactic 445 objects, $X$ and $Y$, leaves $X$ and $Y$ unchanged. The set $\{X, Y\}$ created by MERGE 
446 (Chomsky et al. 2019) cannot be broken and no new features can be added ${ }^{5}$. The

447 original structure in (2a) can be modified by the merging of a new element, $\lambda$, to form

448 (2b), adhering to the No Tampering Condition, while (2c) violates this condition since

449 the original structure (2a) is modified (Lohndal \& Uriagereka 2016); hence why

450 adjuncts that merge 'downstairs' do not alter the structure of the object they adjoin to

451 (adjuncts are not labeled; Bošković 2015), i.e., if 'John saw Mary [in the park]', then

452 the fact that John saw Mary does not change (subscripts denote syntactic

453 heads/labels, standard script denotes lexical items, where (2a) could represent a

454 structure like 'the red boat') ${ }^{6}$.

455

456

457

458

459
(2) a. $\quad[\alpha[\beta[\gamma[\delta \varepsilon]]]]$

b. $\quad[\alpha \lambda[\alpha[\beta[\gamma[\delta \varepsilon]]]]]$

c. $\quad[\alpha[\beta[\gamma[\delta[\varepsilon \varepsilon \lambda]]]]$

Further, it is more economical to expand a structure, as in (2b), than to backtrack and modify a structure that has already been built, as in (2c) (Lasnik \& Lohndal 2013). How

${ }^{5}$ MERGE has been defined as an operation on a workspace and its objects, formalized as follows (WS = workspace; $\mathrm{P} / \mathrm{Q}=$ workspace objects such as linguistic features; $\mathrm{X}=$ additional elements):

$$
\operatorname{MERGE}(P, Q, W S)=W^{\prime}=\left\{\{P, Q\}, X_{1}, \ldots, X_{n}\right\}
$$

Other linguistic frameworks assume some similar, basic structure-building operation, e.g., Forward-Backward Application in Combinatory Categorial Grammar (Steedman 2000), Substitution in Tree-Adjoining Grammar (Joshi \& Schabes 1997). We put aside here some controversies about the relation between a MERGE-based syntax and set theory (see Gärtner 2021).

${ }^{6}$ Adjacent to minimalist syntax, Optimality Theory assumes that gratuitous adjunction to a maximal projection violates an economy condition (SPECLEFT) (Broekhuis \& Vogel 2009, Grimshaw 2001). For syntactic economy conditions in Lexical Functional Grammar, see Dalrymple et al. (2015). 
can we more formally demonstrate these claims? We turn here to Kolmogorov 461 complexity.

\subsection{Compression}

464 Kolmogorov complexity is a measure of the length of the shortest program that can 465 reproduce a given pattern (Kolmogorov 1965, Li \& Vitányi 2019). While measures of minimum description length and Kolmogorov complexity have been typically applied to linear, 'externalized' sequences, they can also be fruitfully applied to grammatical relations, permitting measurement of the inherent information content of an individual object or operation (Biberauer 2019b, Grünwald 1996, 2007, Newmeyer \& Preston

470 2014). Sequence complexity is identified with richness of content (Mitchell 2009), such

471 that any given signal or sequence is regarded as complex if it is not possible to provide

472 a compressed representation of it. While complexity differences across languages can 473 be measured, for example, as a function of featural specifications on functional 474 elements (Longobardi 2017), here we are interested in the complexity of I-language 475 derivations. Previous efforts have already connected the theory of program size to 476 psychology by implementing a concrete language of thought with Turing-computable 477 Kolmogorov complexity (Romano et al. 2013), which satisfied the requirements of (i) 478 being simple enough so that the complexity of any binary sequence can be measured, 479 and (ii) utilizing cognitively plausible operations like printing and repeating. In contrast, 480 we aim to relate similar measures to syntactic economy criteria. relative to other measures of linguistic complexity (Shieber 1985, Trudgill 2011). While 
483 syntacticians have proposed economy principles, these all effectively boil down to 484 efficient tree-search, staying true to basic intuitions like "less search is preferable to 485 more search"-without formalizing these intuitions or attempting to broach this topic 486 with neighboring fields that might be able to provide highly valuable analytic tools.

Syntactic complexity can be operationalized across a number of dimensions, 488 such as online processing/parsing complexity (Hawkins 2004), tree-search and node 489 counts (Szmrecsányi 2004), number of MERGE applications (Samo 2021), 490 cyclic/derivational complexity (Trotzke \& Zwart 2014), internal representational 491 complexity as opposed to derivational size (van Gelderen 2011), entropy reduction 492 (Hale 2016), or stages of second-language development (Walkden \& Breitbarth 2019). 493 Syntactic complexity can be framed as grammar-based (derivational), or user-based 494 (parsing) (Newmeyer \& Preston 2014); here, will be elaborating on the former type. 495 Crucially, sentence length does not always scale with syntactic complexity 496 (Szmrecsányi 2004), and instead an examination of the underlying operations is 497 required. Although syntactic complexity is often thought of in derivational terms, 498 removed and independent from surface realization, Kolmogorov complexity is 499 relatively theory-neutral and can be applied indiscriminately to mental objects with any 500 number of internal sequences, patterns, irregularities, and surface redundancies 501 (Miestamo et al. 2008). Perhaps most importantly, many postulates within theoretical 502 syntax (traditionally grounded in recursive function theory) cannot be obviously derived 503 from independently understood mathematical, physical or biological constructs, 504 whereas the above complexity metrics currently enjoy a more principled relationship 505 with the natural sciences (Ganguli \& Sompolinsky 2012). 
that Kolmogorov complexity (and the related notion of minimal message length) relates

508 directly to frameworks emerging from the FEP (Friston 2019, Hinton \& Zemel 1994,

509 Korbak 2021), with the prediction for natural language syntax of reducing the complexity of hierarchical syntactic structures that are read off at conceptual interfaces

511 being sympathetic to a corollary of the FEP that every computation comes with a 512 concrete energetic cost (Jarzynski 1997, Sengupta \& Stemmler 2014). As shown 513 above (Eq. 2), variational free energy can be formulated as a trade-off between 514 accuracy and complexity, whereby minimising complexity minimises variational free energy. Considering the topic of universal computation, as in Solomonoff induction

516 (Solomonoff 1964) (which is directly grounded in the minimization of Kolmogorov

517 complexity), many formulations of variational free energy minimization explicitly invoke 518 algorithmic complexity and the type of mathematical formulations underlying universal 519 computation. Relating this more directly to our present concerns, the theme of 520 message length reduction has been fruitfully applied to analyses of grammar 521 acquisition in children. Rasin et al. (2021) show that minimum description length 522 (closely related to Bayesian grammar induction) can provide the child with a criterion 523 for comparing hypotheses about grammatical structures that may match basic 524 intuitions across a number of cases. The restrictiveness generated by these complexity measures supplements the more general simplicity criterion of theoretical syntax; much as how the 'subset principle' (restrictiveness) supplemented the original

527 evaluation metric (simplicity) (Berwick 1985). Lambert et al. (2021) demonstrated that 528 the computational simplicity of learning mechanisms appears to have a major impact 529 on the types of patterns found in natural language, including for syntactic trees, and 
so it seems to us well motivated to turn to the issue of the underlying processes that

531 guide the generation of these structures.

Other recent work has successfully used minimum description length in a domain much closer to our own concerns. Focusing on semantic properties of quantifiers (e.g., 'some', 'most') and noting that natural language quantifiers adhere to

535 the properties of monotonicity, quantity and conservativity (Barwise \& Cooper 1981), 536 van de Pol et al. (2021) generated a large collection of over 24,000 logically possible 537 quantifiers and measured their complexity and whether they adhered to the three universal properties. They found that quantifiers that satisfied universal semantic properties were less complex and also exhibited a shorter minimal description length

540 compared to quantifiers that did not satisfy the universals, pointing in intriguing 541 directions towards efficiency biases in natural language semantics that appear to 542 restrict the development of lexical meaning. Quantifiers that adhere to semantic 543 universals are simpler than logically possible competitors that do not. Do these 544 observations also apply at the level of syntactic structure building?

To briefly formalize our discussion of compression and complexity, given a 546 Turing machine $M$, a program $p$ and a string $x$, we can say that the Kolmogorov 547 complexity of $x$ relative to $M$ is defined by the length of $x$. Formally, this can be 548 expressed as follows (Eq. 4), where $|p|$ denotes the length of $p$ and $M$ is any given 549 Turing machine:

$$
K_{M}(x) \stackrel{\text { def }}{=} \min \{|p|: M(p)=x\} \cup\{\infty\}
$$

551 This represents the length of the shortest program that prints the string $x$ and then 552 halts. Yet, as implied by Gödel's incompleteness theorem or Turing's halting theorem, 
553 we cannot compute the Kolmogorov complexity of an arbitrary string, given that it is

554 impossible to test all possible algorithms smaller than the size of the string to be

555 compressed, and given that we cannot know that the Turing machine will halt (Chaitin 556 1995). We therefore used an estimate of approximate Kolmogorov complexity (given

557 its fundamental incomputability) based on the Lempel-Ziv compression algorithm, 558 which we applied to the labeling/search algorithm needed to derive each syntactic 559 node in (2) and their subordinated terminal elements, investigating how 'diverse' the 560 patterns are that are present in any given representation of a syntactic tree-search. In 561 the service of replicability and for completeness, we used a current generative 562 grammar labeling/search algorithm that checks tree-structure heads and terminal 563 elements (Chomsky 2013, Ke 2019, Murphy \& Shim 2020) (see also f.n. 11). In this 564 respect, Kolmogorov complexity is a more fine-grained measure of complexity than 565 previous measures in theoretical syntax (e.g., node count across a c-commanded 566 probe-goal path).

Searching the structure from top to bottom, identifying each branching node 568 and its elements, we used a Lempel-Ziv implementation (Faul 2021) of the classical 569 Kolmogorov complexity algorithm (Kaspar \& Schuster 1987, Lempel \& Ziv 1976) to 570 measure the number of unique sub-patterns when scanning the string of compiled 571 nodes ${ }^{7}$. This Lempel-Ziv algorithm computes a Kolmogorov complexity estimate 572 derived from a limited programming language that permits only copy and insertion in

7 We therefore assume that labeling/search occurs top-to-bottom, and not bottom-up, due to the former yielding a more minimal search path with no 'backtracking', hence more in line with economy considerations. 
573 strings (Kaspar \& Schuster 1987) ${ }^{8}$. The algorithm scans an $n$-digit sequence, $S=s_{1}$.

$574 s_{2} \cdot \ldots s_{n}$, from left to right, and adds a new element to its memory each time it

575 encounters a substring of consecutive digits not previously encountered. Our measure

576 of Kolmogorov complexity takes as input a digital string and outputs a normalised

577 measure of complexity (Urwin et al. 2017).

To connect these ideas with active inference, we note that minimising free

579 energy corresponds to minimising complexity, while maximising the accuracy afforded

580 by internal representations ${ }^{9} r \in R$, of hidden states $s \in S$, given outcomes $o \in O$ (Eq.

581 2). In short, belief updating or making sense of any data implies the minimisation of

582 complexity:

583

$$
D_{K L}[Q(s) \| P(s)] \approx D_{K L}[P(s \mid o) \| P(s)]
$$

584 When choosing how to sample data, the expected complexity becomes the intrinsic 585 value or expected information gain (in expected free energy):

$$
\mathbb{E}[\ln P(s \mid o, \pi)-\ln P(s \mid \pi)]=I(S, O \mid \pi)=\mathbb{E}_{P(o \mid \pi)}\left[D_{K L}[P(s \mid o, \pi) \| P(s \mid \pi)]\right]
$$

587 This is just the mutual information between (unobservable) hidden states generating 588 (observable) outcomes, under a particular choice or policy.

${ }^{8}$ Kaspar and Schuster (1987) discovered that a readily calculable measure of LempelZiv algorithmic complexity can, for simple cellular automata, separate pattern formation from the mere reduction of source entropy, with different types of automata being able to be distinguished. We also note that Lempel-Ziv complexity does not simply measure the number of elements in a sequence, but also factors in pattern irregularities. As such, it is not the case that, by definition, a syntactic process with $n$ steps will be trivially more Lempel-Ziv complex than a syntactic process with $n-1$ steps.

${ }^{9}$ That parameterise posterior beliefs $Q(s) \triangleq Q_{r}(s)$. 
Importantly, variational free energy and formulations of artificial general 590 intelligence pertaining to universal computation both share a mathematical legacy.

591 This is rooted in the relationship between the complexity term in variational free energy 592 and algorithmic complexity (Hinton \& Zemel 1994, Wallace \& Dowe 1999), described 593 in terms of information length and total variation distance. As such, relating syntactic 594 operations to algorithmic compression maximisation feeds directly into assumptions 595 from active inference (Schmidhuber 2010).

Lempel-Ziv complexity is a measure of algorithmic complexity which, under the 597 law of large numbers, plays the same role as the complexity part of log model evidence 598 or marginal likelihood. Interestingly, minimising algorithmic complexity underwrites 599 universal computation, speaking to a deep link between compression, efficiency and 600 optimality in message passing and information processing.

601 With this background, we now return to the structures in (2b) (licensed) and (2c)

602 (unlicensed). Inputting the labeled nodes (subscript elements) and terminal elements 603 (regular script) across both structures into the Lempel-Ziv compression algorithm 604 (Faul 2021) left-to-right, the licensed representation in (2b) exhibits a normalized 605 Kolmogorov complexity of 1.88 , while the unlicensed representation in (2c) exhibits a 606 complexity of 1.99. Crucially, while both (2b) and (2c) exhibit the same node-count 607 complexity and depth (i.e., bracket count), they can be operationally distinguished by 608 their Kolmogorov complexity, in compliance with what the FEP would demand. The 609 increased compression rate for (2b) indicates lower information content, hence lower 
610 Kolmogorov complexity (Juola 2008), and so the representation adheres to the priority

611 to minimise computational load. ${ }^{10}$

612

613

\subsection{Redundancy}

614 Can these types of complexity considerations find a broader architectural basis in

615 theoretical linguistics? Concerns over computational efficiency and representational

616 redundancy motivated the transition beginning in the 1990s from X-Bar models of

617 phrase structure rules involving intermediate hierarchical nodes (e.g., $\left\{\right.$ TP $\left\{N P\left\{T^{\prime}\{T\{V P\right.\right.$

$\left.\left.\left.\left.\left.\left.618\left\{V^{\prime}\{\mathrm{V} N P\}\right\}\right\}\right\}\right\}\right\}\right\}\right)$ to Bare Phrase Structure (BPS), which simply involved set-Merge (e.g.,

$619\{\mathrm{~T}\{\mathrm{NP}\{\mathrm{V}\{\mathrm{T}\{\mathrm{V}\{\mathrm{V}, \mathrm{NP}\}\}\}\}\}\})$. One of the goals of BPS in abandoning indexes, traces and

620 bar-level representations was to minimize the computational burden postulated both

621 within syntax and during the interface of syntax with language-external interpretive

622 systems. To align these themes with our broader concern of processing dynamics

623 (and, hence, biological plausibility), the psycholinguistic validity and polynomial

624 parseability of BPS minimalist grammars has recently been demonstrated (Torr et al.

625 2019). BPS attempts to express parsing stages using the fewest number of possible

626 derivational steps, which is not a concern inherent to other forms of parsers. Both X-

627 Bar and BPS models have been represented with rooted, directed acyclic graphs. The

${ }^{10}$ This example of grounding the No Tampering Condition in a theory-neutral measure of algorithmic complexity relates also to another economy condition in linguistics, the Extension Condition. This states that "Merge always applies in the simplest possible form: at the root" (Chomsky 1995: 248), i.e., there is only one site at which to extend a phrase marker. This condition minimizes complexity and search space, and hence aids compressability of structures fed to the interfaces. 
628 main intuitive difference between X-Bar and BPS concerns when node closure is said 629 to occur, and 'how much' node closure occurs (Stabler 1997, Yngve 1960). In addition, 630 while X-Bar theory permits both binary and unary branching, BPS permits only binary 631 branching; every non-terminal node has exactly two daughters. BPS syntax effectively 632 generates simpler trees, yet with a late-stage burden of evaluating the labeled status 633 of a multi-phrase unit and transferring the constructed workspace to the interfaces 634 (Adger 2003).

635

636

\subsection{Relativised Minimality}

637 A further observation pertaining to economy in the literature concerns Relativised 638 Minimality (Rizzi 1990, 1991): Given a configuration, [X ... Z ... Y], "a local relation 639 cannot connect $X$ and $Y$ if $Z$ intervenes, and $Z$ fully matches the specification of $X$ and $640 Y$ in terms of the relevant features" (Starke 2001). In other words, if $X$ and $Y$ attempt 641 to establish a syntactic relation, but some element, Z, can provide a superset of X's 642 particular features (i.e., X's features plus additional features), this blocks such a 643 relation. In (3a), which game provides a superset of the features hosted by how, 644 resulting in unacceptability. The equivalent does not obtain in $(3 \mathrm{~b})$, and so a 645 relationship between both copies of which game can be established (strikethroughs 646 denote originally merged positions).

647 (3) a. ${ }^{*}\left[\left[\mathrm{How}_{[+\mathrm{Q}]}\right]\left[\mathrm{C}_{[+\mathrm{Q}]}\left[\mathrm{do}\right.\right.\right.$ you wonder $\left[\left[\right.\right.$ which game $\left.{ }_{[+\mathrm{Q},+\mathrm{N}]}\right]\left[\mathrm{C}_{[+\mathrm{Q}]}[\mathrm{PRO}\right.$ to play $648 \quad$ how $[+Q]]]]]]]$ b. $\left[\left[\right.\right.$ Which game $\left.\left.{ }_{[+Q},+N\right]\right]\left[C_{[+Q}\right]$ do you wonder $\left[[\right.$ how $+Q]\left[C_{+Q}[P R O\right.$ to play which game $[+Q,+N]]]]]]]$ 
651 Relativised Minimality emerges directly from minimal search (Aycock 2020): Consider 652 how when searching for matching features in (3b) the search procedure would 'skip' 653 how but find the original copy of which game.

We note that the notion of movement 'distance' here is relativised to the specific units across the path, and so even here formulations of Relativised Minimality seem 656 highly specific to language, as opposed to emerging from some kind of domain657 general constraint. Hence, in order to reach a more fundamental analysis we may 658 need some means of understanding what 'distance' actually reduces to. Under our approach, algorithmic complexity and compressibility may offer a direction forward, potentially being related in some manner to postulates such as syntactic 'phases'. For example, properties of phases, and phase heads, may fall out of independent features

662 of mental computation, perhaps relating to some threshold of compressibility that 663 serves to distinguish one phase from another when assessed alongside representational category. These speculations aside, we can at least conclude for now that there are avenues of current research that lend themselves quite readily to explorations directed by notions of complexity and compression.

\subsection{Resource Restriction}

669 The principle of Resource Restriction (or 'Restrict Computational Resources', RCR; 670 Chomsky 2019, Chomsky et al. 2019) states that when the combinatorial operation 671 MERGE maps workspace $n$ to workspace $n+1$, the number of computationally 672 accessible elements (syntactic objects) can only increase by one (Huybregts 2019, 673 Komachi et al. 2019). This can account for a peculiar property of natural language 
674 recursion that separates it from other forms of recursion (e.g., propositional calculus, 675 proof theory): natural language MERGE involves recursive mapping of workspaces 676 that removes previously manipulated objects (Chomsky 2021c). Hence, Resource 677 Restriction renders natural language derivations strictly Markovian: The present stage 678 is independent of what was generated earlier, unlike standard recursion. Similar 679 observations apply to the idea that when MERGE targets objects in a workspace, non680 targeted elements remain conserved and intact once the new workspace has been 681 established (Chomsky et al. 2019), continuing the intuition of 'No Tampering'. MERGE 682 itself exhibits the formal characteristics of a finite-state rewrite rule (Trotzke \& Zwart 683 2014: 145), exhibiting minimal computational complexity, with MERGE being distinct 684 from the ultimate grammatical constructions later derived from its cyclic application.

A topic of recent discussion concerns how we can define the 'size' of a workspace. Fong et al. (2019) suggest that the size of a syntactic workspace should be considered to be the number of accessible terms plus the number of syntactic objects. This proposal to constrain syntactic combinatorics can account for why the applications of certain types of MERGE are ungrammatical (Fong et al. 2019). This 690 theory also requires only element counting-opening up questions about whether 691 other measures may be used to establish legal operations, such as compressibility. essential once we consider that a workspace with two elements with a simple MERGE

694 operation can generate excessive levels of combinatoriality. Within 8 MERGE steps 695 from two elements, around 8 million distinct sets can be formed (Fong \& Ginsburg 696 2018). Older definitions of basic syntactic computations did not "worry about the fact

697 that it's an organic creature carrying out the activities", as Chomsky (2020) notes. 
698 Many aspects of these theories exhibited, to borrow a phrase from Quine (1995: 5),

699 an "excess of notation over subject matter". Even many current models of syntax have

700 ignored questions of cognitive, implementational plausibility (e.g., Chomsky 2013,

701 Citko \& Gračanin-Yuksek 2021, Collins 2017, Epstein et al. 2022). Computational

702 tractability (van Rooj and Baggio 2021) is a powerful constraint in this respect (e.g.,

703 implementable in polynomial time), and given that minimizing the model complexity

704 term (in formulations of free energy) entails reducing computational cost, this efficiency

705 constraint is also implicitly present in the FEP.

706

707

\subsection{Interim Conclusion}

708 We have considered how the FEP can in principle provide a novel explanation for the 709 prevalence of efficiency-encoded syntactic structures. To further stress this point, consider Dasgupta and Gershman's (2021) assessment that mental arithmetic, mental

711 imagery, planning, and probabilistic inference all share a common resource: memory

712 that enables efficient computation. Other domains exhibiting computational efficiency

713 include concept learning (Feldman 2003), causal reasoning (Lombrozo 2016) and

714 sensorimotor learning (Genewein \& Braun 2014). As Piantadosi (2021) reviews,

715 human learners prefer to induce hypotheses that have a shorter description length in

716 logic (Goodman et al. 2008), with simplicity preferences possibly being "a governing

717 principle of cognitive systems" (Piantadosi 2021: 15; see Chater \& Vitányi 2003).

So far, we have only provided one exemplar case of deriving a component of

719 syntactic design from complexity concerns that relate to formulations of variational free 720 energy minimisation. We will now turn to the most commonly explored syntactic 
721 processes claimed to arise from economy considerations: syntactic movement and 722 minimal search. Further examples will be used to motivate what we term the principle 723 of Turing-Chomsky Compression, through which stages of syntactic derivations are

724 evaluated based on the algorithmic compressibility of some feature of the computation, such as the movement path of an object, or the procedure of nodal labeling/searchwhich can be unified based on how they manipulate the syntactic workspace.

\section{Minimising Free-Energy, Maximising Interpretability}

As has long been recognised, the syntactic categories of words are not tagged acoustically, and yet sentential meaning is inferred from syntactic categorization

731 (Adger 2019). Interpreting thematic relations (who did what to whom) demands that relations between words are established, however spoken sentences are often ambiguous between distinct syntactic structures. For instance, below we can interpret

734 Jim Carrey as starring in the movie (4a), or sitting next to us (4b).

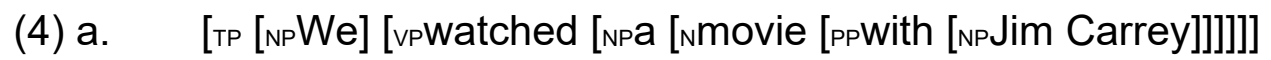
in a sentence) can be contrasted with structural distance (the number of hierarchical

739 nodes intervening), and only the latter is a significant predictor of reading times in an 740 eye-tracking corpus (Baumann 2014). Violations of hierarchical sentence rules results 741 in slower reading times (Kush et al. 2015), and expectations of word category based 742 on hierarchical grammars also predicts reading times (Boston et al. 2011). 
The apparent use of hierarchical structure to limit interpretation adheres to a

744 core tenet of the FEP, whereby interpretive processes that yield the lowest possible

745 amount of complexity (and thereby computational cost) can mostly (perhaps entirely;

746 Hinzen 2006) be derived directly from what the syntactic component produces. This

747 notion is closely related to the imperatives for structure learning (Tervo et al. 2016) -

748 or Bayesian model reduction-in optimising the structural (syntax) of generative

749 models based purely on complexity (pertaining to model parameters); see Friston, Lin

750 et al. (2017) for an example simulating active inference and insight in rule learning.

$751 \quad$ While sensorimotor systems naturally impose linear order, linguistic 752 expressions are complex $n$-dimensional objects with hierarchical relations (Gärtner \& 753 Sauerland 2007, Grohmann 2007, Kosta et al. 2014, Murphy 2016). The following 754 sections provide concrete demonstrations of these design principles in action.

\subsection{Structural Distance}

757 Consider the sentence in (5).

758

(5) Routinely, poems that rhyme evaporate.

759 In (5), 'routinely' exclusively modifies 'evaporate'. The matrix predicate 'evaporate' is 760 closer in terms of structural distance to 'routinely' than to 'rhyme', since the relative 761 clause embeds 'rhyme' more deeply (minimal search is partly "defined by least 
762

763

embedding"; Chomsky 2004: 109) ${ }^{11}$. Language computes over structural distance, not linear distance (Berwick et al. 2011, 2013, Friederici et al. 2017, Martin et al. 2020). This can also be shown with simple interrogative structures. Consider the sentence in (6a) and its syntactic representation in (6b), where the verb in the relative clause ('rhyme') is more deeply embedded than 'evaporate'.

(6) a. Do poems that rhyme evaporate?

b. $\quad[\mathrm{CP}[\mathrm{C}$ Do][TP[DP[DP poems $][\mathrm{CP}[\mathrm{C}$ that $][\mathrm{TP}$ rhyme $]][\mathrm{T}[\mathrm{T}][\mathrm{v}$ evaporate $]]]]$

We can compute the complexity of both nodal search and Kolmogorov complexity, contrasting the grammatical association between 'Do' and 'evaporate', and the ungrammatical association between 'Do' and 'rhyme'. When the $[+Q]$ feature on $C$ searches for a goal, it needs to search down three node steps (from CP to V) to get to the grammatical option, but needs to search down four node steps (from CP to embedded TP) to construct the ungrammatical option. Since we are concerned with analyzing a small but representative number of syntactic derivational processes, this analysis differed from the approach to the structures in (2), which did not involve any

${ }^{11}$ We refer the reader to Ke (2019: 44) and Aycock (2020: 3-6) for a detailed discussion of minimal search, which can be formally defined below, from Aycock (2020), adopting an Iterative Deepening Depth-First Search approach (Korf 1985); where MS = minimal search, $\mathrm{SA}=$ search algorithm, SD = search domain (where SA operates), ST = search target:

(1) $\mathrm{MS}=\langle\mathrm{SA}, \mathrm{SD}, \mathrm{ST}\rangle$

(2) $S A$ :

a. Given ST and SD, match against every head member of SD to find ST [initial depth-limit of $S D=1$; search depth-first].

b. If ST is found, return the head(s) bearing ST and go to d. Otherwise, go to c.

c. Increase the depth-limit of SD by 1 level; return to a.

d. Terminate Search. 
777 labeling procedure. This time, we enumerated the search steps across nodes, 778 replacing specific nodal categories with symbols interpretable to the Lempel-Ziv 779 compression algorithm (Faul 2021), since this is what the syntactic search algorithm 780 is claimed to monitor. The Lempel-Ziv complexity of the sequence of steps 781 enumerated from the C-V labeling/search algorithm is 1.72 . For the embedded C-TP 782 search, it increases to 2.01.

Predictions about grammaticality generated by tree-search depth and 784 Kolmogorov complexity estimates such as Lempel-Ziv may provide an additional 785 advantage to models of syntax that attempt to establish these (and potentially other) forms of sympathies with active inference, relative to rival theories. For instance, while one might invoke purely semantic constraints on polar interrogatives and other forms of question-formation (Bouchard 2021) to derive the kinds of acceptability contrasts we have discussed, we see no way to ground these observations in concerns of computability and complexity, and no way to measure or formalize these notions.

As recent literature has explored, whenever there is a conflict between principles of computational efficiency and principles of communicative clarity, the former seems to be prioritized (Asoulin 2016, Murphy 2020a). For instance, consider (7).

797 The individual ('Saul') and the object ('car') can be questioned, but questioning the 798 more deeply embedded object forces the speaker to produce a more complex 799 circumlocution ('[ ]' denotes the originally merged position of the wh-expression). 
(8) a. $\quad{ }^{*}[$ What $]$ did you persuade who to sell [ ]?

801

b. [Who] did you persuade [ ] to sell what?

802 The structures in (8) involve the same words and interpretations, yet the more computationally costly process of searching for-and then moving — the more deeply embedded element cannot be licensed, despite the potential benefits of 805 communicative flexibility. Interestingly, one cannot feasibly posit parsing-related 806 factors to derive some independent complexity measure to explain this contrast (e.g., 807 Newmeyer 2007), given the same number of words and same semantic interpretations 808 (i.e., give me the Agent and Object of the event). Experimental work has supported 809 the prevalence of these grammaticality intuitions (Clifton et al. 2006). Crucially, this is 810 not to say that when language is used for communication that this process is not also 811 structured via criteria of efficiency; for instance, see Gibson et al. (2019) (but see also 812 Galantucci et al. 2020 for evidence that people often fail to-and, indeed, do not care 813 to-communicate faithfully). Rather, we aim to stress the existence of conflicting goals 814 of economy. ${ }^{12}$ Consider also a model of dialogue through which internal generative models 816 used to infer one's own behaviour are deployed to infer the communicative intentions 817 of another, given both parties have similar generative models (Friston \& Frith 2015a). 818 As such, the core notion of surprise might be a crucial adjudicator in framing the

12 We also highlight here the generalisation, discussed extensively in Jackendoff and Wittenberg (2014), that simpler syntactic structures typically lead to a greater reliance on pragmatics for successful communication, whereas larger sentences lead to more of an interpretive burden being placed on syntactic principles instead of conversational context. 
819 distinction between I-language and more social conceptions of language production:

820 Reducing surprise/effort internally serves I-language functions of computational

821 efficiency, but it can also serve to encourage mutual predictability between speakers,

822 while, on the other hand, intentionally inducing surprise in others can often directly

823 serve certain communicative goals (Giorgi \& Dal Farra 2019).

824

The syntactic structures for both (8a) and (8b) are represented in (9) (where

$825<\mathrm{DP}>$ represents the movement path). With respect to tree-search depth, (9a) involves

826 searching down 11 nodes, while (9b) involves searching down 9 nodes. To expand

827 our survey of syntactic processes beyond labeling/search paths, we focused here on

828 the postulated path of syntactic object movement across the structure. The movement

829 path was represented with each site being attributed a symbol fed to the compression

830 algorithm, in keeping with a more general approach to annotating movement paths

831 (Adger 2003). Enumerating the movement path from the initially merged root, to

832 intermediate landing sites, to the terminal landing site in Spec-CP, the Lempel-Ziv

833 complexity of movement for $(9 a)$ is 2.15 . For $(9 b)$, path complexity is 1.5 .

834

(9) a. [CP [DP what] [C [c did] [TP [DP you] [T' [T [pres] [VP [<DP>] [V' [v persuade] [CP

$\left[\mathrm{c}^{\prime}[\mathrm{c} \varnothing][\mathrm{TP}[<\mathrm{DP}>][\mathrm{T},[\mathrm{T}][\mathrm{VP}[\mathrm{DP}\right.$ who] [pP [P to] [VP [v' [v buy] [<DP> $\left.\left.\left.\left.\left.\left.\left.\left.\left.\left.]]]]\right]\right]\right]\right]\right]\right]\right]\right]\right]\right]$

836

b. $\quad[\mathrm{CP}[\mathrm{DP}$ who] [c' [c did] [TP [DP you] [T, [T [pres] [VP [<DP>] [V' [v persuade] [CP $\left[\mathrm{C}^{\prime}[\mathrm{c} \varnothing]\left[\mathrm{TP}[<\mathrm{DP}>]\left[\mathrm{T}^{\prime}[\mathrm{T}][\mathrm{vP}[<\mathrm{DP}>][\mathrm{PP}[\mathrm{P}\right.\right.\right.$ to] [VP [v' [v buy] [DP what]]]]]]]]]]]]]]]

838 A further empirical reason to assume that this economy condition is a general property

839 of language comes from the following data of Bulgarian multiple wh-fronting (Bošković 840 \& Messick 2017; see also Dayal 2017 for discussion of the Superiority Condition). The 841 wh-phrase highest prior to movement (the subject in (10) and the indirect object in

842 (11)) needs to be first in the linear order of the sentence, such that the structurally 
843 highest wh-phrase moves first, and the second wh-phrase either right-adjoins to the

844 first wh-phrase, or moves to a structurally lower Spec-CP position.

845

846

847

848

849

850

851

852

853

854

855

856

857

858

859

860

861

862

863

864 865

a. ${ }^{*}$ Koj e vidjal kogo?

who is seen whom

b. Koj kogo e vidjal?

"Who saw whom?"

a. Kogo kakvo e pital Ivan? whom what is asked Ivan "Whom did Ivan ask what?"

b. *Kakvo kogo e pital Ivan?

Thus far, this suffices to show that the wh-element easiest to search for is selected for movement. However, does syntactic economy simply rule out all but one option? Crucially, Bošković and Messick (2017) show that when multiple options of equal treegeometric complexity are available, they are both licensed as grammatical. Consider constructions with three wh-phrases. We can assume that whichever wh-element moves to the structurally highest position (Spec-CP) satisfies the featural requirement of interrogative $\mathrm{C}$ to have a filled Spec-CP position. After this structurally highest element moves to Spec-CP, we can further assume that the remaining wh-elements then move to Spec-CP to satisfy their own featural 'Focus'-based requirements. At this point, whichever order the remaining wh-elements move in, the requirements are satisfied through movements of identical length (i.e., both cross the same number of nodes, and hence generate the same sequence of derivational steps, and therefore the same Lempel-Ziv complexity). As such, this predicts that the remaining two wh- 
elements can move in any order after the initial wh-movement of the subject. This

867 prediction is borne out: the subject ('koj') is moved first in both constructions below, 868 but then either of the remaining wh-elements can move in any order.

869

a. Koj kogo kakvo e pital?

870 who whom what is asked

871 "Who asked whom what?"

872 b. Koj kakvo kogo e pital?

873

874

\subsection{Subject-Auxiliary Inversion}

875 Another use of minimal tree-search to derive acceptability dynamics is found in the 876 observation that MERGE cannot select the linearly closest auxiliary in English Subject877 Auxiliary inversion, but must search for the structurally closest auxiliary.

878 (13) Somebody who is in Texas is on the phone.

879

880

a. * ${ }^{\text {Is }] ~ s o m e b o d y ~ w h o ~[~] ~ i n ~ T e x a s ~ i s ~ o n ~ t h e ~ p h o n e ? ~}$

b. [Is] somebody who is in Texas [ ] on the phone?

881 In (14b), the search algorithm is a depth of 3 (moving from the matrix CP to the 882 immediately embedded VP, 'is on the phone', and finding its root auxiliary), while in 883 (14a) the search is a depth of 4 (from the matrix CP to the immediately embedded 884 relative clause, to the VP, and then its root auxiliary). Mapping the search path of (14a) 885 yields a complexity of 2. Mapping the search path of (14b), which is the same as (14a) 886 minus the intermediate relative clause, yields a complexity of 1.58 . 


\subsection{Labeling}

889 As a more stringent test, can Lempel-Ziv complexity shed light on cases in which the 890 ungrammatical derivation has less structural tree-geometric complexity than the 891 grammatical derivation? Consider the following case from Murphy and Shim (2020: 892 204). Putting ancillary technical details aside (see Mizuguchi 2019, Murphy \& Shim 893 2020), (15a) is claimed to be ungrammatical because one final necessary operation 894 on the syntactic workspace has not been carried out; namely, merging 'the students' 895 to the structure marked by $\mathrm{y}$. For expository purposes, we provide a schematic 896 representation to demonstrate the relevant movement path (the path of 'the student' 897 is marked by $t$ ).

898

899

a. *[y Seems to be likely [a the student [to [t understand the theory]]]] b. [o The student [y seems to be likely [a $t$ [to [ $t$ understand the theory]]]]]

The explanations from within syntactic theory as to why $(15 b)$ is grammatical concern successful feature valuation and the minimal search of copies via the labeling algorithm. However, this process might also be linked to more efficient compression rates of syntactic labels at the interpretive systems. We can enumerate each labeled 904 node left-to-right marking the phrase boundaries separating each embedded object that pertain to the grammaticality contrast (e.g., V-D-P-V). Computing the Lempel-Ziv complexity of each successive phrase label in these structures, (15a) exhibits a 907 complexity of 1.86 , while (15b) exhibits a complexity of 1.66 , despite (15b) being a 908 more complex structure from the perspectives of node count and element count. As 909 such, both minimal search of syntactic labels and algorithmic compression rates may be playing separate but related roles in determining how the interpretive systems 911 access objects generated by syntax. 


\subsection{Turing-Chomsky Compression}

914 The brief number of exemplars we have derived syntactic economy principles from, 915 using a Lempel-Ziv estimate of Kolmogorov complexity, motivate the following 916 principle of language design:

Turing-Chomsky Compression: An operation $(M)$ on an accessible object $\left(O_{1}\right)$ in a syntactic workspace $\left(W_{p}\right)$ minimizes variational free energy if structures from the resulting workspace $\left(W_{q}\right)$ are compressed to a lower Kolmogorov complexity than if $M$ had accessed $\mathrm{O}_{2}$ in $W_{p}$.

921 This is principally named after specifications over what (Chomsky) is compressed and 922 how (Turing) such compression can be achieved (Chomsky 2021c, Turing 1950). The 923 interaction between Turing-Chomsky Compression (TCC) and more domain-specific 924 subcategorization requirements emerging from lexico-semantic features, and formal 925 syntactic features, is a promising topic for future research. We have shown across a 926 small but representative number of syntactic processes that derivations minimising 927 algorithmic complexity are licensed over those that result in structures and derivational 928 paths that are less compressible. In keeping with TCC, the examples we presented 929 constitute preliminary evidence that operations on syntactic workspaces are evaluated 930 by criteria that is independent of language-specific representational features-a step 931 towards a "genuine explanation" (Chomsky 2022) for language design.

\section{Future Work}



out to be a brief and rare spark in the universe, one that we may soon extinguish. We are seeking to understand what may be a true marvel of the cosmos."

938 We have arrived at a number of suggestive explanations for the way language 939 implements the construction of hierarchical syntactic objects: to minimise uncertainty 940 about the causes of sensory data, and to adhere to a least effort natural law (i.e.,

941 variational principle of least action), when composing sets of linguistic features for 942 interpretation, planning, prediction and externalization. We have shown that 943 measuring a Kolmogorov complexity estimate of syntactic representations and 944 movement paths can align with acceptability judgments. This was used to motivate a 945 new principle of language design, Turing-Chomsky Compression (TCC). Our use of 946 Lempel-Ziv complexity presents a more explicit measure than previous accounts. For 947 instance, consider Sternefeld's (1997) Global Economy Condition, which states that, 948 given two derivations of a syntactic structure (D1, D2), D1 is preferred if D1 fares better 949 than D2 with respect to some metrical measure M (namely, number of derivational 950 steps). This basic 'step counting' measure (as with tree-search depth) seems to be in 951 line with grammaticality predictions of the more general complexity measure provided 952 by Lempel-Ziv complexity. Yet, Lempel-Ziv complexity also benefits from being 953 applicable across a range of other domains in syntax where nodal count does not differ 954 between competing structures, and is also related to formulations of variational free 955 energy minimization. Ultimately, this has the advantage of generating quantitative 956 predictions for syntactic computation based on general principles that apply more 957 broadly. 

et al. 2021), one could feasibly view our research programme as comparing the

960 information length of belief updating between distinct syntactic derivations and 961 theories. We view our proposals as being, in principle, concordant with the view that neural representations in organic agents evolve by approximating steepest descent in information space towards the point of optimal inference (Da Costa et al. 2021). Future work could explore the utility of minimum description length (van de Pol et al. 2021) and Gell-Mann/Lloyd 'effective complexity'. In contrast to Kolmogorov complexity, which measures the description length of a whole object, effective complexity measures the description length of regularities (structured patterns) within an object (Gell-Mann \& Lloyd 1996), which may speak to properties of cyclic, phasal computation in natural language.

More recent work has provided evidence for a mental compression algorithm in

971 humans (termed the Language of Thought chunking algorithm) responsible for parsing 972 very basic, binary sequences, providing evidence that human sequence coding 973 involves a form of internal compression using language-like nested structures (Planton 974 et al. 2021). Dehaene et al. (2022) extend this project to auditory sequences and 975 geometrical shapes. We have effectively extended these ideas further into the domain 976 of natural language syntax, suggesting some common capacity for symbolic recursion 977 across cognitive systems being constrained by compressibility. While hierarchical complexity in language has clear neurobiological correlates 979 and energetic costs involved that relate to entropy and surprisal measures (Brennan 980 et al. 2016), the neurobiological status of more specific components of syntax that 981 pertain to economy criteria-such as the dynamics of BPS parsing-remains much 
982 less clear. As a means to establish more direct, and testable connections between 983 these aspects of syntax and neighbouring domains of the cognitive sciences, we have 984 used a small number of exemplar cases to demonstrate that Lempel-Ziv complexity 985 (i) can align with nodal distance measures; (ii) can provide accurate predictions for 986 legal syntactic objects when nodal distance is identical across legal and illegal 987 syntactic objects; and (iii) can implement a principle of free energy minimization. In 988 addition, Lempel-Ziv complexity has been used to successfully measure the 989 complexity of cortical activity across a number of domains, including sleep and 990 wakefulness (Abásolo et al. 2015), the quantification of spikes and bursts of synchronization (Blanc et al. 2008), the aging brain (Shumbayawonda et al. 2018), cortical complexity under general anesthesia (Puglia et al. 2021), and the reduction of resting-state neural complexity in schizophrenia relative to neurotypical controls 994 (Fernández et al. 2011). For linguists, we believe that the complexity metrics we have 995 discussed can constitute supporting hypotheses that connect theories of grammar to 996 observable psychophysical measurements and neural signals.

Some linguists might object to our complexity measure in the following way: 998 Why should syntax be organized so as to produce structures that minimise 999 Kolmogorov complexity, and why should the semantic component of language aim to 1000 read off structures that are of corresponding complexity? We note in response that the 1001 core 'phase'/non-phase pattern of syntactic derivations (e.g., $\{C\{T \vee\{\vee D / n\{N\}\}\}$; 1002 Richards 2011, Uriagereka 2012) optimizes compression rate (effectively, 010101), 1003 and since phase construction constitutes the major determining period when syntactic 1004 workspaces are accessed by the conceptual systems, we see our proposal as aligning 1005 closely with existing —if only implicit-assumptions. 
theory to model natural language syntax (Coopmans et al. 2021) have—-thus far-only modeled binary set-formation and lexical selection criteria without using the tools of category theory to explain the design of these syntactic structures, economy criteria, or other more complex syntactic features such as displacement. While category theory

1011 is functionally useful for contrasting language with other cognitive faculties such as 1012 action (as shown in Coopmans et al. 2021), the separate goal—and our present goal— 1013 of grounding apparent economy constraints in syntax within a biologically plausible 1014 model of language and computation in neural systems, and how these complex 1015 systems implement these constraints, may require other formal tools.

1017 need to be further unpacked and clarified. Our proposals concerning compression of 1018 structures accessed from the syntactic workspace via TCC have been discussed in 1019 the context of a cursory overview of the mathematical lineage shared between 1020 formulations pertaining to the FEP and theories of universal computation. This suited 1021 our current expository purposes, with our proposals being buttressed by conceptual 1022 overviews of the FEP and syntactic economy, but future work should more carefully 1023 align models of active inference with TCC. Although we made our assumptions about syntactic complexity based on whether or not our measure can be formally grounded within the FEP, we note that we have effectively equated complexity with compressibility. As such, we acknowledge that there may be a number of other fruitful

1027 directions to measure complexity in ways that are sympathetic to active inference (e.g., 1028 the "complexity equals change" framework; Aksentijevic \& Gibson 2012). 
1030 proposals, reflecting internal aspects of parser states, with entropy measuring their

1031 uncertainty and relating to expected payoffs, and Kolmogorov complexity measuring

1032 their compressibility (indeed, Shannon's entropy is an upper bound on Kolmogorov

1033 complexity). For instance, Yun et al. (2015) combine minimalist syntax with an entropy

1034 reduction complexity metric to derive word-by-word predictions across Chinese,

1035 Japanese and Korean. Looking at different types of relative clauses (subject relative

1036 and object relative) that involve different syntactic movement paths, their entropy

1037 reduction pattern directly matches independent psycholinguistic measures of reading

1038 difficulty. Conceptually speaking, entropy reduction measures and Kolmogorov

1039 complexity measures speak to the costs of endogenous syntactic computations, being

1040 more directly informative about internal states than more externalist measures like

1041 surprisal (Murphy 2021).

A challenge for future research is to appropriately frame the interfacing of

1043 syntax with other systems in terms that accord with the minimisation of surprise and 1044 variational free energy. Since the FEP has attendant process theories (e.g., active 1045 inference), one of the latent payoffs of our suggestions here is the development of 1046 generative models of active inference that fully ground specific factors in syntactic 1047 theory and, through simulation work, may align with recent advances in the 1048 electrophysiology, neural dynamics and neural harmonics of syntax (Keitel et al. 2017, 1049 Tavano et al. 2021). For example, one crucial factor in any syntax model seems to be 1050 phrasal category information, since it appears to drive cortical tracking of hierarchical 1051 structures (Benítez-Burraco \& Murphy 2019, Burroughs et al. 2021, Murphy 2020b, 1052 Murphy, Hoshi et al. 2022, Wilkinson \& Murphy 2016), in line with work highlighting 
NATURAL LANGUAGE SYNTAX COMPLIES WITH THE FREE-ENERGY PRINCIPLE

1053 the unique computational contribution of phrase structure labeling (Adger 2013, 1054 Hornstein 2009, Murphy 2015b, Zhao 2021).

1055

1056

\section{Conclusions}

1057 We have reviewed how the FEP, that underwrites active inference, is an expression 1058 of the principle of least action, which is additionally a principle implemented in models 1059 of theoretical syntax. The FEP provides suggestions for why the brain computes the 1060 way it does, while theoretical linguistics provides a means of establishing what is 1061 computed. Our discussion of this topic culminated in a novel design principle for 1062 natural language, Turing-Chomsky Compression (TCC). An intuition from 70 years 1063 ago-that the extent to which a model of grammar is simple seems to determine its explanatory power-echoes in the modern framing of syntactic computation as a system of economy: "[T]he motives behind the demand for economy are in many ways the same as those behind the demand that there be a system at all" (Chomsky 1951; 1067 see also Goodman 1951). For Rissanen (1989), "given some data, the simplest 1068 hypothesis explaining it is preferable". Generative linguistics has long appealed to 1069 economy considerations (e.g., the evaluation metric in Chomsky \& Halle 1968). This appeal to simplicity may even emerge from an evolved bias shared across our species

1071 to favour simple solutions (see the 'cognitive simplicity hypothesis'; Terzian \& 1072 Corbalán 2021). The FEP has produced formal, simulation-supported models of 1073 complex cognitive mechanisms such as action, perception, learning, attention and 1074 communication, while theories of syntax embracing computational efficiency have led

1075 to empirically successful outcomes, explaining grammaticality intuitions (Adger 2003, 1076 Martin et al. 2020, Sprouse 2011, Sprouse \& Almeida 2017), certain poverty of 
1077 stimulus issues (Berwick et al. 2011, Crain et al. 2017, Culbertson et al. 2012, Wexler 1078 2003, Yang et al. 2017) and the pervasive organizational role that hierarchy has in 1079 language (Friederici et al. 2017, Grimaldi 2012) and the seemingly unique proclivity 1080 humans have to parse sequences into tree-structures.

Our approach also mirrors previous applications of the FEP to cognition. 1082 Exploring the issue of subgoaling, Maisto et al. (2015) conclude that favoured 1083 subgoals are ones that permit planning solutions and controlling behaviour using fewer 1084 information resources (yielding parsimony in inference and control), and conclude that 1085 of those strings that represent procedures returning the same output, strings with lower descriptive complexity are more probable, and should more likely be selected by 1087 probabilistic inference. They argue that this framing is compatible with the FEP, and 1088 we have further argued here that these assumptions of computational efficiency in 1089 subgoals can be found in syntactic combinatorics (e.g., Momma \& Phillips 2018 1090 discuss linguistic parsing and production in terms of discrete subgoals). We find seeds 1091 for these ideas in the foundational principles of universal computation, where, as we 1092 have noted, free energy is often discussed in terms of minimum description or 1093 message lengths (MacKay 2003, Schmidhuber 2010). Relatedly, core findings from 1094 dependency length minimization (DLM) research suggest that, during online parsing, comprehenders seek to minimize the total length of dependencies in a given structure since this reduces working memory load (Gibson et al. 2019). These overt, 'visible' 1097 and linearized relations may therefore be constrained by similar efficiency criteria of 1098 the kind we have discussed here, which seem to shape the operations of syntactic 1099 structure building. 
1101 is the way it is (Adger 2019, Narita 2014), and we have suggested that the FEP can

1102 contribute to this goal. The more efficiently a language user can internally construct 1103 meaningful, hierarchically organized syntactic structures, the more readily they can 1104 use these structures to contribute to the planning and organization of action, reflection 1105 and consolidation of experience, exogenous and endogenous monitoring, imagination 1106 of possible states, adaptive environmental sampling, and the consideration of personal 1107 responsibilities. We used a brief number of examples to demonstrate proof of concept 1108 for how compression algorithms, such as a Kolmogorov complexity estimate, can provide principled insight into efficiency concerns alongside more traditional economy criteria such as node count and tree-search depth. Given that one can characterize major components of mental life as being derived from information compression (e.g., 1112 casting aside the vast majority of perceptual experience that does not inform generative models), our conclusion that the most basic component of natural language executes the same process as a primary constraint on its activities and its interfacing with other cognitive systems should be seen as closely sympathetic to algorithmic 1116 information theories of cognition and consciousness (Ruffini 2017). Other potential 1117 topics to explore include Case systems of natural language, which do not seem to be 1118 motivated purely by semantic demands (Chomsky 2020, 2021c) and which seem 1119 instead to help the facilitation of perception and parsing by establishing clear relations 1120 between discourse elements. How this and other components of natural language 1121 relate to the priorities of active inference is a promising direction to pursue. Indeed, it 1122 may transpire that developing a general understanding of computational efficiency for 1123 organic, mental systems may only be possible by adopting an interdisciplinary, 
1124 systematic approach to natural phenomena (Cipriani 2021), of the kind afforded by the

1125 active inference framework, moving beyond the types of descriptions initially tailored 1126 specifically to linguistic categories.

Using examples involving minimal search and syntactic movement, we have

1128 shown that natural language syntax and its interfacing with conceptual systems is 1129 uniformly constrained by rates of algorithmic compression, motivating what we term 1130 the TCC principle, which may be a foundational organizing constraint on language design. While linguists can speak of the 'Superiority Condition', the 'labeling algorithm' and 'minimal search', we have claimed that some (perhaps all) of these postulates can 1133 be derived from the goal of minimizing variational free energy via TCC. From the 1134 perspective of active inference, individuals need to minimise the effort involved in 1135 meaning-making, and TCC contributes to this goal. Active inference assumes an 1136 imperative to find the most accurate explanation for sensory observations that is 1137 minimally complex-recruited in Barlow's (2001) exploration of minimum 1138 redundancy-and which seems in accord with how the language system builds 1139 structure, and structural relations (Hauser \& Watumull 2017).

We note in this context recent work by van Gelderen $(2018,2021)$. Using a 1141 series of historical reviews of extensive language changes, van Gelderen (2021) 1142 shows that syntactic economy principles can account for language variation across a 1143 broad variety of phenomenon, including that-trace constructions, CP-deletion, and the 1144 presence of expletives. In addition, van Gelderen (2018) argues that regular patterns 1145 of language change can be seen as resolutions to labeling failures (i.e., when the 1146 labeling/search algorithm 'fails' to label a given structure). This work reveals how 1147 considerations of economy (that we have argued arise directly from active inference) 
1148 are not simply formal stipulations useful in accounting for grammaticality-they can 1149 also yield principled insights into large-scale dynamics in historical language change.

1150 Moving beyond, we note that our measures of Kolmogorov complexity in the 1151 operations of natural language syntax are exploiting a highly general, theory-neutral measure of complexity, and that these and other related measures serve to index 1153 some underlying, independent process of organic computation, which remains elusive 1154 in its formal character and neural basis. While some domain-specificity will continue 1155 to be required in the language sciences, if only due to the closed class of lexical objects that syntax accesses, we hope to have shown that theory-neutral measures of complexity that have previously been related to formulations of the FEP can provide new inroads for the study of language.

More broadly, what the FEP can offer theoretical linguistics is proof of principle:

a foundational grounding and means of additional motivation for investigating 1161 language in terms of efficient computation. The FEP is fundamentally a normative 1162 model (Allen 2018) which can aid the generation of implementational/process models 1163 (such as predictive coding or active inference) and can place constraints on feasibility. 1164 Further simulation and modeling work is required to push these ideas further for natural language and its various sub-systems, and we envisage that this type of future work will provide fruitful insights into natural language syntax. If this project can be expanded in this manner, we could make significant gains in ways that traditional 1168 experimental psycholinguistics and neuroimaging of language processing have often been unable to-we may discover a means to reconcile universal properties of human language syntax with endogenous properties of the brain. 
NATURAL LANGUAGE SYNTAX COMPLIES WITH THE FREE-ENERGY PRINCIPLE

1172 Open Practices Statement: No empirical data is associated with this article.

\section{References}

1174 Abásolo, D., Simons, S., da Silva, R.M., Tononi, G., \& Vyazovskiy, V.V. (2015). Lempel-Ziv complexity 1175 of cortical activity during sleep and waking in rats. Journal of Neurophysiology, 113(7), 27422752.

1177 Adger, D. (2003). Core Syntax: A Minimalist Approach. Oxford: Oxford University Press.

Adger, D. (2013). A Syntax of Substance. Cambridge, MA: MIT Press.

1179

Adger, D. (2019). Language Unlimited: The Science Behind Our Most Creative Power. Oxford: Oxford University Press.

Adger, D., \& Svenonius, P. (2011). Features in minimalist syntax. In C. Boeckx (Ed.), The Oxford Handbook of Linguistic Minimalism (pp. 27-51). Oxford: Oxford University Press.

Aksentijevic, A., \& Gibson, K. (2012). Complexity equals change. Cognitive Systems Research, 15-16, $1-16$.

Al-Mutairi, F.R. (2014). The Minimalist Program: The Nature and Plausibility of Chomsky's Biolinguistics. Cambridge: Cambridge University Press.

Allen, M. (2018). The foundation: mechanism, prediction, and falsication in Bayesian enactivism. Comment on Answering Schrödinger's question: a free-energy formulation, by Maxwell James Desormeau Ramstead et al. Physics of Life Reviews, 24, 17-20.

Andrews, M. (2021). The math is not the territory: navigating the free energy principle. Biology \& Philosophy, 36, 30.

1192 Asoulin, E. (2016). Language as an instrument of thought. Glossa: A Journal of General Linguistics, 1(1), 46 .

Aycock, S. (2020). A third-factor account of locality: explaining impenetrability and intervention effects with minimal search. Cambridge Occasional Papers in Linguistics, 12(1), 1-30. 
NATURAL LANGUAGE SYNTAX COMPLIES WITH THE FREE-ENERGY PRINCIPLE

1196

1197

1198

1199

1200

1201

1202

1203

1204

1205

1206

1207

1208

1209

1210

1211

1212

1213

1214

1215

1216

1217

1218

1219

Barandiaran, X.E., \& Chemero, A. (2009). Animats in the modeling ecosystem. Adaptive Behavior, 17(4), 287-292.

Barlow, H. (1961). Possible principles underlying the transformations of sensory messages. In W. Rosenblith (Ed.), Sensory Communication (pp. 217-234). Cambridge, MA: MIT Press.

Barlow, H.B. (1974). Inductive inference, coding, perception, and language. Perception, 3, 123-134.

Barlow, H. (2001). Redundancy reduction revisited. Computation and Neural Systems, 12, 241-253.

Barwise, J., \& Cooper, R. (1981). Generalized quantifiers and natural language. Linguistics and Philosophy, 4(2), 159-219.

Bastos, A. M., Martin Usrey, W., Adams, R. A., Mangun, G. R., Fries, P., Friston, K. J. (2012). Canonical microcircuits for predictive coding. Neuron, 76, 695-711.

Baumann, P. (2014). Dependencies and hierarchical structure in sentence processing. Proceedings of CogSci 2014, 152-157.

Benítez-Burraco, A., \& Murphy, E. (2019). Why brain oscillations are improving our understanding of language. Frontiers in Behavioral Neuroscience, 13, 190.

Berwick, R.C. (1985). The Acquisition of Syntactic Knowledge. Cambridge, MA: MIT Press.

Berwick, R.C., Friederici, A.D., Chomsky, N., \& Bolhius, J.J. (2013). Evolution, brain, and the nature of language. Trends in Cognitive Sciences, 17(2), 89-98.

Berwick, R.C., Pietroski, P., Yankama, B., \& Chomsky, N. (2011). Poverty of the stimulus revisited. Cognitive Science, 35(7), 1207-1242.

Berwick, R.C., \& Stabler, E.P. (Eds). (2019). Minimalist Parsing. Oxford: Oxford University Press.

Biberauer, T. (2019a). Factors 2 and 3: towards a principled approach. Catalan Journal of Linguistics Special Issue, 45-88.

Biberauer, T. (2019b). Some thoughts on the complexity of syntactic complexity. Theoretical Linguistics, 45(3-4), 259-274. 
NATURAL LANGUAGE SYNTAX COMPLIES WITH THE FREE-ENERGY PRINCIPLE

1220

1221

1222

1223

1224

1225

1226

1227

1228

1229

1230

1231

1232

1233

1234

1235

1236

1237

1238

1239

1240

1241

1242

1243

1244

Blanc, J-L., Schmidt, N., Bonnier, L., Pezard, L., \& Lesne, A. (2008). Quantifying neural correlations using Lempel-Ziv complexity. Deuxième conférence française de Neurosciences Computationnelles, Neurocomp08. Marseille, France.

Bobaljik, J. (2002). A-chains at the PF-interface: copies and covert movement. Natural Language \& Linguistic Theory 20(2): 197-267.

Bošković, Ž. (2015). From the complex NP constraint to everything: on deep extractions across categories. The Linguistic Review, 32, 603-669.

Bošković, Ž. (2016). On the timing of labeling: deducing Comp-trace effects, the Subject Condition, the Adjunct Condition, and tucking in from labeling. The Linguistic Review, 33, 17-66.

Bošković, Ž. (2021). Merge, move, and contextuality of syntax: the role of labeling, successive-cyclicity, and EPP effects. Ms. University of Connecticut.

Bošković, Ž., \& Lasnik, H. (Eds). (2007). Minimalist Syntax: The Essential Readings. Oxford: Blackwell.

Bošković, Ž., \& Messick, T. (2017). Derivational economy in syntax and semantics. In M. Aronoff (Ed.), Oxford Research Encyclopedia of Linguistics. Oxford: Oxford University Press.

Boston, M., Hale, J., Vasishth, S., \& Kliegl, R. (2011). Parallel processing and sentence comprehension difficulty. Language and Cognitive Processes, 26, 301-349.

Bouchard, D. (2021). Three conceptions of nativism and the faculty of language. Languages Sciences, 85,101384

Bouizegarene, N., Ramstead, M., Constant, A., Friston, K., \& Kirmayer, L. (2020). Narrative as active inference. PsyArXiv. https://doi.org/10.31234/osf.io/47ub6.

Bourguignon, M., Molinaro, N., Lizarazu, M., Taulu, S., Jousmäki, V., Lallier, M., Carreiras, M., \& Tiège, X.D. (2020). Neocortical activity tracks the hierarchical linguistic structures of self-produced speech during reading aloud. Neurolmage, 216, 116788.

Brennan, J.R., \& Martin, A.E. (2020). Phase synchronization varies systematically with linguistic structure composition. Philosophical Transactions of the Royal Society B, 375(1791), 20190305. 
NATURAL LANGUAGE SYNTAX COMPLIES WITH THE FREE-ENERGY PRINCIPLE

1245

1246

1247

1248

1249

1250

1251

1252

1253

1254

1255

1256

1257

1258

1259

1260

1261

1262

1263

1264

1265

1266

1267

1268

1269

Brennan, J.R., Stabler, E.P., Van Wagenen, S.E., Luh, W-M., \& Hale, J.T. (2016). Abstract linguistic structure correlates with temporal activity during naturalistic comprehension. Brain and Language, 157-158, 81-94.

Broekhuis, H., \& Vogel, R. (Eds.) (2009). Optimality Theory and Minimalism: Interface Theories. Potsdam: Universitätsverlag Potsdam.

Brown, H., Adams, R. A., Parees, I., Edwards, M., \& Friston, K. (2013). Active inference, sensory attenuation and illusions. Cognitive Processing, 14(4), 411-427.

Burroughs, A., Kazanina, N., \& Houghton, C. (2021). Grammatical category and the neural processing of phrases. Scientific Reports, 11, 2446.

Cardinaletti, A., \& Starke, M. (1999). The typology of structural deficiency: a case study of the three classes of pronouns. In Henk van Riemsdijk (Ed.), Clitics in the Languages of Europe (pp. 145233). New York: Mouton de Gruyter.

Chaitin G.J. (1995). Randomness in arithmetic and the decline and fall of reductionism in pure mathematics. In J. Cornwell (Ed.), Nature's Imagination (pp. 27-44). Oxford: Oxford University Press.

Chater, N., \& Vitányi, P. (2003). Simplicity: a unifying principle in cognitive science? Trends in Cognitive Sciences, 7(1), 19-22.

Chomsky N. (1949). Morphophonemics of Modern Hebrew. Undergraduate Honors Thesis, University of Pennsylvania, Philadelphia.

Chomsky, N. (1956). Three models for the description of language. IRE Transactions on Information Theory, 2, 113-124.

Chomsky, N. (1959). On certain formal properties of grammars. Information and Control, 2, 137-167.

Chomsky N. (1951/1979). Morphophonemics of Modern Hebrew. New York: Garland.

Chomsky, N. (1986). Knowledge of Language: Its Nature, Origin, and Use. Westport, CT: Praeger.

Chomsky, N. (1995). The Minimalist Program. Cambridge, MA: MIT Press. 
NATURAL LANGUAGE SYNTAX COMPLIES WITH THE FREE-ENERGY PRINCIPLE

1270

1271

1272

1273

1274

1275

1276

1277

1278

1279

1280

1281

1282

1283

1284

1285

1286

1287

1288

1289

1290

1291

1292

1293

Chomsky, N. (2000). New Horizons in the Study of Language and Mind. Cambridge: Cambridge University Press.

Chomsky, N. (2004). Beyond explanatory adequacy. In A. Belletti (Ed), Structures and Beyond (pp. 104-131). Oxford: Oxford University Press.

Chomsky, N. (2008). On phases. In R. Freidin, C.P. Otero, \& M.L. Zubizaretta (Eds.), Foundational Issues in Linguistic Theory: Essays in Honor of Jean-Roger Vergnaud (pp. 133-166). Cambridge, MA: MIT Press.

Chomsky, N. (2011). Language and other cognitive systems. What is special about language? Language, Learning and Development, 7(4), 263-278.

Chomsky, N. (2013). Problems of projection. Lingua, 130, 33-49.

Chomsky, N. (2014). Minimal recursion: exploring the prospects. In T. Roeper \& M. Speas (Eds.), Studies in Theoretical Psycholinguistics 43. Recursion: Complexity in Cognition (pp. 1-15). London: Springer.

Chomsky, N. (2019). Some puzzling foundational issues: the Reading Program. Catalan Journal of Linguistics Special Issue, 263-285.

Chomsky, N. (2020). Minimalism: where we are now and where we are going. Talk given at the Linguistic Society of Japan. 22 November.

Chomsky, N. (2021a). Linguistics then and now: some personal reflections. Annual Review of Linguistics, 7, 1-11.

Chomsky N. (2021b). Simplicity and the form of grammars. Journal of Language Modelling, 9(1), 5-15.

Chomsky, N. (2021c). Minimalism: where we are now, and where we can hope to go. Gengo Kenkyu (Journal of the Linguistic Society of Japan), 160, 1-41.

Chomsky, N. (2022). Genuine explanation and the strong minimalist thesis. Lecture at the Biolinguistics Fall Semester class, University of Arizona, 24 August. 
NATURAL LANGUAGE SYNTAX COMPLIES WITH THE FREE-ENERGY PRINCIPLE

1294 Chomsky, N., Gallego, Á.J., \& Ott, D. (2019). Generative grammar and the faculty of language: insights, questions, and challenges. Catalan Journal of Linguistics Special Issue, 226-261.

1296

Chomsky, N., \& Halle, M. (1968). The Sound Pattern of English. New York: Harper and Row.

1297

Cipriani, E. (2021). On Chomsky's notion of explanatory adequacy. Language and Linguistics Compass

1298 15(11): e12441.

1299

Citko, B., \& Gračanin-Yuksek, M. (2021). Merge: Binarity in (Multidominant) Syntax. Cambridge, MA:

1300 MIT Press.

1301

Clifton, C., Fanselow, G., \& Frazier, L. (2006). Amnestying superiority violations: processing multiple 1302 questions. Linguistic Inquiry, 37(1), 51-68.

1303

Collins, C. (2017). Merge $(X, Y)=\{X, Y\}$. In L. Bauke \& A. Blümel (Eds.), Labels and Roots, pp. 47-68. De 1304 Gruyter.

1305

Collins, J. (2020). Conjoining meanings without losing our heads. Mind \& Language, 35, 224-236.

1306

Colombo, M., \& Wright, C. (2021). First principles in the life sciences: the free-energy principle, 1307 organicism, and mechanism. Synthese, 198, 3463-3488.

1308

Coopersmith, J. (2017). The Lazy Universe: An Introduction to the Principle of Least Action. Oxford: Oxford University Press.

1310

Coopmans, C.W., Kaushik, K., \& Martin, A.E. (2021). Hierarchical structure in language and action: a formal comparison. PsyArXiv doi:10.31234/osf.io/x59un.

1312 Crain, S., Koring, L., \& Thornton, R. (2017). Language acquisition from a biolinguistic perspective. Neuroscience and Biobehavioral Reviews, 81(B), 120-149.

1314 Culbertson, J., Smolensky, P., \& Legendre, G. (2012). Learning biases predict a word order universal. Cognition, 122(3), 306-329.

1316 Da Costa, L., Parr, T., Sengupta, B., \& Friston, K. (2021). Neural dynamics under active inference: plausibility and efficiency of information processing. Entropy, 23, 454. 
NATURAL LANGUAGE SYNTAX COMPLIES WITH THE FREE-ENERGY PRINCIPLE

1318

1319

1320

1321

1322

1323

1324

1325

1326

1327

1328

1329

1330

1331

1332

1333

1334

1335

1336

1337

1338

1339

1340

1341

1342

1343

Dalrymple, M., Kaplan, R.M., \& King, T.H. (2015). Economy of expression as a principle of syntax. Journal of Language Modelling, 3(2), 377-412.

Dasgupta, I., \& Gershman, S.J. (2021). Memory as a computational resource. Trends in Cognitive Sciences, 25(3), 240-251.

Dayal, V. (2017). Multiple wh-questions. In M. Everaert \& H.C. Riemsdijk. The Wiley Blackwell Companion to Syntax (Second Edition). Oxford: Blackwell.

Dehaene, S., Al Roumi, F., Lakretz, Y., Planton, S., \& Sablé-Meyer, M. (2022). Symbols and mental programs: a hypothesis about human singularity. Trends in Cognitive Sciences, 26(9), 751-766.

Dobashi, N. (2010). Computational efficiency in the syntax-phonology interface. The Linguistic Review, 27(3), 241-260.

Embick, D., \& Poeppel, D. (2015). Towards a computational(ist) neurobiology of language: correlational, integrated, and explanatory neurolinguistics. Language, Cognition and Neuroscience, 30(4), 357-366.

Epstein, S.D., Groat, E., Kawashima, R., \& Kitahara, H. (1998). A Derivational Approach to Syntactic Relations. Oxford: Oxford University Press.

Epstein, S.D., Kitahara, H., \& Seely, T.D. (2021). A Minimalist Theory of Simplest Merge. New York: Routledge.

Evans, D.J., \& Searles, D.J. (1994). Equilibrium microstates which generate second law violating steady states. Physical Review E, 50, 1645-1648.

Faul, S. (2021). Kolmogorov complexity, MATLAB Central File Exchange. Retrieved November 23, 2021.

Feldman, J. (2003). The simplicity principle in human concept learning. Current Directions in Psychological Science, 12(6), 227-232.

Fernández, A., López-Ibor, M-I., Turrero, A., Santos, J-M., Morón, M-D., Hornero, R., Gómez, C., Méndez, M.A., Ortiz, T., \& López-Ibor, J.J. (2011). Lempel-Ziv complexity in schizophrenia: a MEG study. Clinical Neurophysiology, 122(11), 2227-2235. 
NATURAL LANGUAGE SYNTAX COMPLIES WITH THE FREE-ENERGY PRINCIPLE

1344

1345

1346

1347

1348

1349

1350

1351

1352

1353

1354

1356

1357

1358

1359

1360

1361

1362

1363

1364

1365

1366

1367 1368

Feynman, R.P. (1972). Statistical Mechanics. Reading, MA: Benjamin.

Fong, S., Berwick, R.C., \& Ginsburg, J. (2019). The combinatorics of Merge and workspace right-sizing. Paper presented at Evolinguistics Workshop, 25-26 May.

Fong, S., \& Ginsburg, J. (2018). On constraining Free Merge. The 43rd Meeting of the Kansai Linguistics Society. Konan University: Kobe, Japan.

Fox, D. (2000). Economy and Semantic Interpretation. Cambridge, MA: MIT Press.

Frampton, J., \& Gutmann, S. (1999). Cyclic computation, a computationally efficient minimalist syntax. Syntax, 2(1), 1-27.

Friederici, A.D., Chomsky, N., Berwick, R.C., Moro, A., \& Bolhuis, J.J. (2017). Language, mind and brain. Nature Human Behaviour, 1, 713-722.

Freidin, R., \& Lasnik, H. (2011). Some roots of minimalism. In C. Boeckx (Ed.), The Oxford Handbook of Linguistic Minimalism (pp. 1-26). Oxford: Oxford University Press.

Friston, K. (2010). The free-energy principle: a unified brain theory? Nature Reviews Neuroscience, 11(2), 127-138.

Friston, K. (2013). Life as we know it. Journal of the Royal Society Interface, 10, 20130475.

Friston, K.J. (2019). Complexity and computation in the brain: The knowns and the known unknowns. In W. Singer, T.J. Sejnowski, \& P. Rakic (Eds.), The Neocortex (pp. 269-291). Cambridge, MA: MIT Press.

Friston, K., FitzGerald, T., Rigoli, F., Schwartenbeck, P., \& Pezzulo, G. (2017). Active inference: a process theory. Neural Computation, 29(1), 1-49.

Friston, K.J., \& Frith, C.D. (2015a). A duet for one. Consciousness and Cognition, 36, 390-405.

Friston, K.J., \& Frith, C.D. (2015b). Active inference, communication and hermeneutics. Cortex, 68, $129-143$.

Friston, K.J., Lin, M., Frith, C.D., Pezzulo, G., Hobson, J.A., \& Ondobaka, S. (2017). Active inference, curiosity and insight. Neural Computation, 29(10), 2633-2683. 
NATURAL LANGUAGE SYNTAX COMPLIES WITH THE FREE-ENERGY PRINCIPLE

1369 Friston, K.J., Parr, T., \& de Vries, B. (2017). The graphical brain: belief propagation and active inference. Network Neuroscience, 1(4), 381-414.

1371 Friston, K.J., Parr, T., Yufik, Y., Sajid, N., Price, C.J., \& Holmes, E. (2020). Generative models, linguistic communication and active inference. Neuroscience and Biobehavioral Reviews, 118, 42-64.

Friston, K.J., Rosch, R., Parr, T., Price, C., \& Bowman, H. (2017). Deep temporal models and active inference. Neuroscience \& Biobehavioral Reviews, 77, 388-402.

Friston, K.J., Sajid, N., Quiroga-Martinez, D.R., Parr, T., Price, C.J., \& Holmes, E. (2021). Active listening. Hearing Research, 399, 107998.

1377

Fukui, N. (1996). On the nature of economy in language. Cognitive Studies, 3, 51-71.

1378

Fuster, J.M. (2004). Upper processing stages of the perception-action cycle. Trends in Cognitive Sciences, 8(4), 143-145.

Galantucci, B., Langsteim, B., Spivack, E., \& Paley, N. (2020). Repair avoidance: when faithful informational exchanges don't matter that much. Cognitive Science, 44(10), e12882.

1382

Gallego, Á.J., \& Chomsky, N. (2020). The faculty of language: a biological object, a window into the 1383 mind, and a bridge across disciplines. Revista de la Sociedad Española de Lingüística, 50(1), 734.

Gallego, Á.J., \& Martin, R. (Eds). (2018). Language, Syntax and the Natural Sciences. Cambridge: Cambridge University Press.

Gallego, Á.J., \& Orús, R. (2022). Language design as information renormalization. SN Computer Science, 3, 140. 1-27.

Ganguli, S., \& Sompolinsky, H. (2012). Compressed sensing, sparsity, and dimensionality in neuronal information processing and data analysis. Annual Review of Neuroscience, 35, 485-508.

1391 Gärtner, H.-M. (2021). Copies from "standard set theory"? A note on the foundation of minimalist syntax 1392 in reaction to Chomsky, Gallego and Ott (2019). Journal of Logic, Language and Information. https://doi.org/10.1007/s10849-021-09342-x. 
NATURAL LANGUAGE SYNTAX COMPLIES WITH THE FREE-ENERGY PRINCIPLE

1394

1395

1396

1397

1398

1399

1400

1401

1402

1403

1404

1405

1406

1407

1408

1409

1410

1411

1412

1413

1414 Grimaldi, M. (2012). Toward a neural theory of language: old issues and new perspectives. Journal of 1415

1416

1417 1418

Gärtner, H.-M., \& Sauerland, U. (Eds.). (2007). Interfaces + Recursion = Language? Chomsky's Minimalism and the View from Syntax-Semantics. Berlin: De Gruyter Mouton.

Genewein, T., \& Braun, D.A. (2014). Occam's razor in sensorimotor learning. Proceedings of the Royal Society B, 281(1783), 2013-2952.

van Gelderen, E. (2011). The Linguistic Cycle: Language Change and the Language Faculty. Oxford: Oxford University Press.

van Gelderen, E. (2018). Problems of projection: the role of language change in labeling paradoxes. Studia Linguistica, 72, 113-127.

van Gelderen, E. (2021). Third Factors in Language Variation and Change. Cambridge: Cambridge University Press.

Gell-Mann, M., \& Lloyd, S. (1996). Information measures, effective complexity, and total information. Complexity, 2(1), 44-52.

Gibson, E., Futrell, R., Piantadosi, S.P., Dautriche, I., Mahowald, K., Bergen, L., \& Levy, R. (2019). How efficiency shapes human language. Trends in Cognitive Sciences, 23(5), 389-407.

Giorgi, A., \& Dal Farra, C. (2019). On the syntax/pragmatics interface: expressing surprise and disapproval. Intercultural Pragmatics, 16(3), 335-361.

González Escribano, J.L. (2005). Semantocentric minimalist grammar. Atlantis, 27(2), 57-74.

Goodman N. (1951). The Structure of Appearance. Cambridge, MA: Harvard University Press.

Goodman, N., Tenenbaum, J., Feldman, J., \& Griffiths, T. (2008). A rational analysis of rule-based concept learning. Cognitive Science, 32(1), 108-154. Neurolinguistics, 25(5), 304-327.

Grimshaw, J. (2001). Economy of structure in OT. Rutgers Optimality Archive 444.

Grohmann, K. (Ed.). (2007). InterPhases: Phase-Theoretic Investigations of Linguistic Interfaces. Oxford: Oxford University Press. 
NATURAL LANGUAGE SYNTAX COMPLIES WITH THE FREE-ENERGY PRINCIPLE

1419 Grünwald, P. (1996). A minimum description length approach to grammar inference. In S. Wermter, E. 1420 Riloff, \& G. Scheler (Eds.), Connectionist, Statistical and Symbolic Approaches to Learning for Natural Language Processing. IJCAI 1995. Lecture Notes in Computer Science (Lecture Notes in Artificial Intelligence), vol 1040 (pp. 203-216). Berlin: Springer.

1423 Grünwald, P.D. (2007). The Minimum Description Length Principle. New York: MIT Press.

1424

1425

1426

1427

1428

1429

1430

1431

1432

1433

1434

1435

1436

1437

1438

1439

1440

1441

1442

1443

Haegeman, L. (1994). Introduction to Government and Binding Theory. 2nd edition. Oxford: Blackwell.

Hale, J.T. (2016). Information-theoretical complexity metrics. Language and Linguistics Compass, 10, $397-412$.

Hauser, M., Chomsky, N., \& Fitch, W.T. (2002). The faculty of language: what is it, who has it, and how did it evolve? Science, 298(5598), 1569-1579.

Hauser, M., \& Watumull, J. (2017). The universal generative faculty: the source of our expressive power in language, mathematics, morality, and music. Journal of Neurolinguistics, 43(B), 78-94.

Hawkins, J. (2004). Efficiency and Complexity in Grammars. Oxford: Oxford University Press.

Heim, I., \& Kratzer, A., (1998). Semantics in Generative Grammar. Oxford: Blackwell.

Hinton, G.E., \& Zemel, R.S. (1994). Autoencoders, minimum description length and Helmholtz free energy. In J.D. Cowan, G. Tesauro, \& J. Alspector, (Eds.), Advances in Neural Information Processing Systems (pp. 3-10). San Mateo, CA: Morgan Kaufmann.

Hinzen, W. (2006). Mind Design and Minimal Syntax. Oxford: Oxford University Press.

Hinzen, W. (2016). On the grammar of referential dependence. Studies in Logic, Grammar and Rhetoric, 46(1), 11-33.

Hinzen, W., \& Sheehan, M. (2013). The Philosophy of Universal Grammar. Oxford: Oxford University Press.

Hohwy, J. (2016). The self-evidencing brain. Noûs, 50, 259-285.

Hohwy, J. (2017). How to entrain your evil demon. In T. Metzinger \& W. Wiese (Eds.), Philosophy and Predictive Processing 2 (pp. 1-15). Frankfurt am Main: MIND Group. 
NATURAL LANGUAGE SYNTAX COMPLIES WITH THE FREE-ENERGY PRINCIPLE

1444

1445

1446

1447

1448

1449

1450

1451

1452

1453

1454

1455

1456

1457

1458

1459

1460

1461

1462

1463

1464

1465

1466

1467

1468

1469

Hohwy, J. (2020). New directions in predictive processing. Mind \& Language, 35(2), 209-223.

Holmberg, A., \& Roberts, I. (2014). Parameters and the three factors of language design. In C. Picallo (Ed.). Linguistic Variation in the Minimalist Framework (pp. 61-81). Oxford: Oxford University Press.

Hornstein, N. (2009). A Theory of Syntax: Minimal Operations and Universal Grammar. Cambridge: Cambridge University Press.

Hornstein, N., \& Pietroski, P. (2009). Basic operations: minimal syntax-semantics. Catalan Journal of Linguistics, 8, 113-139.

Hutter, M. (2006). Universal Artificial Intellegence: Sequential Decisions Based on Algorithmic Probability. Dordrecht: Springer-Verlag Berlin and Heidelberg \& Co. KG.

Huybregts, M.A.C. (2019). Infinite generation of language unreachable from a stepwise approach. Frontiers in Psychology, 10, 425.

Jackendoff, R., \& Wittenberg, E. (2014). What you can say without syntax: a view from evolution. In F.J. Newmeyer \& L.B. Preston (Eds.). Measuring Grammatical Complexity (pp. 65-82). Oxford: Oxford University Press.

Jarzynski, C. (1997). Nonequilibrium equality for free energy differences. Physical Review Letters, 78(14), 2690-2693.

Joshi, A.K., \& Schabes, Y. (1997). Tree-adjoining grammars. In G. Rozenberg \& A. Salomaa (Eds.), Handbook of Formal Languages: Volume 3 Beyond Words (pp. 69-123). Berlin, Heidelberg: Springer.

Juola, P. (2008). Assessing linguistic complexity. In M. Miestamo, K. Sinnemaki \& F. Karlsson (Eds.), Language Complexity: Typology, Contact, Change (pp. 89-107). Amsterdam: Benjamins.

Kaspar, F., \& Schuster, H.G. (1987). Easily calculable measure for the complexity of spatiotemporal patterns. Physical Review A, 36(2), 842-848.

Katzir, R., O'Donnell, T.J., \& Rasin, E. (2021). Introduction to the special issue on simplicity in grammar learning. Journal of Language Modeling, 9(1), 1-4. 
NATURAL LANGUAGE SYNTAX COMPLIES WITH THE FREE-ENERGY PRINCIPLE

$1470 \mathrm{Ke}, \mathrm{H}$. (2019). The Syntax, Semantics and Processing of Agreement and Binding Grammatical Illusions.

$1471 \quad \mathrm{PhD}$ dissertation. University of Michigan.

1472 Keitel, A., Ince, R.A.A., Gross, J., \& Kayser, C. (2017). Auditory cortical delta-entrainment interacts with 1473 oscillatory power in multiple fronto-parietal networks. Neurolmage, 147, 32-42.

1474 Kirchhoff, M., Parr, T., Palacios, E., Friston, K., \& Kiverstein, J. (2018). The Markov blankets of life: autonomy, active inference and the free energy principle. Journal of the Royal Society Interface, 15(138), 20170792.

1477 Kirchhoff, M. D., \& Robertson, I. (2018). Enactivism and predictive processing: a non-representational view. Philosophical Explorations, 21(2), 264-281.

1479

Kleene, S.C. (1952). Introduction to Metamathematics. New York: Van Nostrand.

1480

Kolmogorov, A.N. (1965). Three approaches to the quantitative definition of information. Problems of Information Transmission, 1, 1-7.

Komachi, M., Kitahara, H., Uchibori, A., \& Takita, K. (2019). Generative procedure revisited. Reports of 1483 the Keio Institute of Cultural and Linguistic Studies, 50, 269-283.

1484 Korbak, T. (2021). Computational enactivism under the free energy principle. Synthese, 198, 27431485 2763.

1486 Korf, R. (1985). Depth-first iterative-deepening: an optimal admissable tree search. Artificial 1487 Intelligence, 27(1), 97-109.

1488 Kosta, P., Schürcks, L., Franks, S., \& Radev-Bork, T. (Eds.). (2014). Minimalism and Beyond: 1489 Radicalizing the Interfaces. Amsterdam: John Benjamins.

1490 Kush, D., Lidz, J., \& Phillips, C. (2015). Relation-sensitive retrieval: evidence from bound variable 1491 pronouns. Journal of Memory and Language, 82, 18-40.

1492 Lambert, D., Rawski, J., \& Heinz, J. (2021). Typology emerges from simplicity in representations and 1493 learning. Journal of Language Modelling, 9(1), 151-194.

1494 Larson, B. (2015). Minimal search as a restriction on merge. Lingua, 156, 57-69. 
NATURAL LANGUAGE SYNTAX COMPLIES WITH THE FREE-ENERGY PRINCIPLE

1495 Lasnik, H, \& Lohndal, T. (2013). Brief overview of the history of generative syntax. In M. den Dikken 1496 (Ed.). The Cambridge Handbook of Generative Syntax (pp. 26-60). Cambridge: Cambridge $1497 \quad$ University Press.

1498 Leivada, E., \& Murphy, E. (2021). Mind the (terminological) gap: 10 misused, ambiguous, or 1499 polysemous terms in linguistics. Ampersand, 8, 100073.

1500 Lempel, A., \& Ziv, J. (1976). On the complexity of finite sequences. IEEE Transactions on Information 1501 Theory, 22(1), 75-81.

1502 Li, M., \& Vitányi, P. (2019). An Introduction to Kolmogorov Complexity and Its Applications. 4th edition. $1503 \quad$ New York: Springer.

1504 Linsker, R. (1990). Perceptual neural organization: some approaches based on network models and 1505 information theory. Annual Review of Neuroscience, 13, 257-281.

1506

Lobina, D.J. (2017). Recursion: A Computational Investigation into the Representation and Processing 1507 of Language. Oxford: Oxford University Press.

1508 Lohndal, T., \& Uriagereka, J. (2016). Third-factor explanations and universal grammar. In I. Roberts 1509 (Ed.). The Oxford Handbook of Universal Grammar (pp. 114-128). Oxford: Oxford University Press.

1511 Lombrozo, T. (2016). Explanatory preferences shape learning and inference. Trends in Cognitive Sciences, 20(10), 748-759.

1513 Longobardi, G. (2008). Reference to individuals, person, and the variety of mapping parameters. In A. Klinger \& H. Mueller (Eds.), Essays on Nominal Determination: From Morphology to Discourse Management (pp. 189-211). Amsterdam: John Benjamins.

Longobardi, G. (2017). Principles, parameters, and schemata: A radically underspecified UG. Linguistic Analysis, 41(3-4), 517-558.

1518 Lupyan, G., \& Clark, A. (2015). Words and the world: predictive coding and the language-perceptioncognition interface. Current Directions in Psychological Science, 24(4), 279-284. 
NATURAL LANGUAGE SYNTAX COMPLIES WITH THE FREE-ENERGY PRINCIPLE

1520

1521

1522

1523

1524

1525

1526

1527

1528

1529

1530

1531

1532

1533

1534

1535

1536

1537

1538

1539

1540

1541

1542

1543

1544

MacGregor, J.N. (1987). Short-term-memory capacity: limitation or optimization? Psychological Review, 94(1), 107-108.

MacKay, D.J. (1995). Free-energy minimisation algorithm for decoding and cryptoanalysis. Electronics Letters, 31, 445-447.

MacKay, D.J. (2003). Information Theory, Inference, and Learning Algorithms. Cambridge: Cambridge University Press.

Maisto, D., Donnarumma, F., \& Pezzulo, G. (2015). Divide et impera: subgoaling reduces the complexity of probabilistic inference and problem solving. Journal of the Royal Society Interface, 12(104), 20141335.

Martin, A., Holtz, A., Abels, K., Adger, D., \& Culbertson, J. (2020). Experimental evidence for the influence of structure and meaning on linear order in the noun phrase. Glossa: A Journal of General Linguistics, 5(1), 97.

Miestamo, M., Sinnemäki, K., \& Karlsson, F. (Eds). (2008). Language Complexity: Typology, Contact, Change. Amsterdam: Benjamins.

Mikhail, J. (2007). Universal moral grammar: theory, evidence and the future. Trends in Cognitive Sciences, 11(4), 143-152.

Ming, L., \& Vitányi, P. (2008). An Introduction to Kolmogorov Complexity and Its Applications. 3rd ed. New York: Springer.

Mitchell, M. (2009). Complexity: A Guided Tour. Oxford: Oxford University Press.

Mizuguchi, M. (2019). Optional raising in ECM and labeling of XP-YP. Generative Grammar Research, 29(2), 373-411.

Murphy, E. (2015a). Labels, cognomes, and cyclic computation: an ethological perspective. Frontiers in Psychology, 6, 715.

Murphy, E. (2015b). Reference, phrases and individuation: topics at the labeling-interpretive interface. Opticon1826, 17, 5. 
NATURAL LANGUAGE SYNTAX COMPLIES WITH THE FREE-ENERGY PRINCIPLE

1545

1546

1547

1548

1549

1550

1551

1552

1553

1554

1555

1556

1557

1558

1559

1560

1561

1562

1563

1564

1565

1566

1567

1568

1569

Murphy, E. (2016). Phasal eliminativism, anti-lexicalism, and the status of the unarticulated. Biolinguistics, 10, 21-50.

Murphy, E. (2020a). Language design and communicative competence: the minimalist perspective. Glossa: A Journal of General Linguistics, 5(1), 2.

Murphy, E. (2020b). The Oscillatory Nature of Language. Cambridge: Cambridge University Press.

Murphy, E. (2021). The citadel itself: defending semantic internalism. PsyArXiv doi:10.31234/osf.io/czqm5.

Murphy, E., \& Shim, J-Y. (2020). Copy invisibility and (non-)categorial labeling. Linguistic Research, 37(2), 187-215.

Murphy, E., Hoshi, K., \& Benítez-Burraco, A. (2022). Subcortical syntax: reconsidering the neural dynamics of language. Journal of Neurolinguistics, 62, 101062.

Murphy, E., Woolnough, O., Rollo, P.S., Roccaforte, Z., Segaert, K., Hagoort, P., \& Tandon, N. (2022). Minimal phrase composition revealed by intracranial recordings. Journal of Neuroscience https://doi.org/10.1523/JNEUROSCI.1575-21.2022.

Narita, H. (2014). Endocentric Structuring of Projection-free Syntax. Amsterdam: John Benjamins.

Newmeyer, F.J. (2007). More complicated and hence, rarer: a look at grammatical complexity and crosslinguistic rarity. In S. Karimi, V. Samiian \& W.K. Wilkins (Eds.), Clausal and Phrasal Architecture: Syntactic Derivation and Interpretation (Festschrift for Joseph E. Emonds) (pp. 221242). Amsterdam: John Benjamins.

Newmeyer, F.J., \& Preston, L.B. (Eds.) (2014). Measuring Grammatical Complexity. Oxford: Oxford University Press.

Palacios, E.R., Razi, A., Parr, T., Kirchhoff, M., \& Friston, K. (2020). On Markov blankets and hierarchical self-organization. Journal of Theoretical Neurobiology, 486, 110089.

Parr, T., Da Costa, L., \& Friston, K. (2020). Markov blankets, information geometry and stochastic thermodynamics. Philosophical Transactions of the Royal Society A, 378(2164), 20190159. 
NATURAL LANGUAGE SYNTAX COMPLIES WITH THE FREE-ENERGY PRINCIPLE

1570

1571

1572

1573

1574

1575

1576

1577

1578

1579

1580

1581

1582

1583

1584

1585

1586

1587

1588

1589

1590

1591

1592

1593

1594

Parr, T., \& Friston, K.J. (2017). Working memory, attention, and salience in active inference. Scientific Reports, 7(1), 14678.

Parr, T., \& Friston, K.J. (2019). Attention or salience? Current Opinion in Psychology, 29, 1-5.

Pearl, J. (1988). Probabilistic Reasoning in Intelligent Systems: Networks of Plausible Inference. San Francisco, CA: Morgan Kaufmann.

Penny, W.D., Stephan, K.E., Mechelli, A., \& Friston, K.J. (2004). Comparing dynamic causal models. Neurolmage, 22(3), 1157-1172.

Peterson, T. (2016). Mirativity as surprise: evidentiality, information, and deixis. Journal of Psycholinguistic Research, 45(6), 1327-1357.

Piantadosi, S.T. (2021). The computational origin of representation. Minds and Machines, 31, 1-58.

Pietroski, P. (2005). Events and Semantic Architecture. Oxford: Oxford University Press.

Pietroski, P. (2018). Conjoining Meanings: Semantics Without Truth Values. Oxford: Oxford University Press.

Planton, S., van Kerkoerle, T., Abbih, L., Maheu, M., Meyniel, F., Sigman, M., Wang, L., Figueira, S., Romano, S., \& Dehaene, S. (2021). A theory of memory for binary sequences: evidence for a mental compression algorithm in humans. PLoS Computational Biology, 17(1), e1008598.

van de Pol, I., Lodder, P., van Maanen, L., Steinert-Threlkeld, S., \& Szymanik, J. (2021). Quantifiers satisfying semantic universals are simpler. PsyArXiv. June 4. doi:10.31234/osf.io/xuhyr.

Puglia, M.P., Li, D., Leis, A.M., Jewell, E.S., Kaplan, C.M., Therrian, M., Kim, M., Lee, U., Mashour, G.A., \& Vlisides, P.E. (2021). Neurophysiologic complexity in children increases with developmental age and is reduced by general anesthesia. Anesthesiology, 135, 813-828.

Pulvermüller, F. (2014). The syntax of action. Trends in Cognitive Sciences, 18(5), 219-220.

Quine, W.V.O. (1995). Whitehead and the rise of modern logic. Selected Logic Papers (pp. 1-36). Cambridge, MA: Harvard University Press.

Radford, A. (2016). Analysing English Sentences. Cambridge: Cambridge University Press. 
NATURAL LANGUAGE SYNTAX COMPLIES WITH THE FREE-ENERGY PRINCIPLE

1595

1596

1597

1598

1599

1600

1601

1602

1603

1604

1605

1606

1607

1608

1609

1610

1611

1612

1613

1614

1615

1616

1617

1618

1619

Rahman, M.S., \& Kaykobad, M. (2005). On Hamiltonian cycles and Hamiltonian paths. Information Processing Letters, 94, 37-41.

Ramchand, G., \& Svenonius, P. (2014). Deriving the functional hierarchy. Language Sciences, 46, $152-$ 174.

Ramstead, M. J. D., Badcock, P. B., \& Friston, K. J. (2018). Answering Schrödinger's question: A freeenergy formulation. Physics of Life Reviews, 24, 1-16.

Ramstead, M.J., Kirchhoff, M.D., Constant, A., \& Friston, K.J. (2021). Multiscale integration: beyond internalism and externalism. Synthese, 198, 41-70.

Rao, R.P., \& Ballard, D.H. (1999). Predictive coding in the visual cortex: a functional interpretation of some extra-classical receptive-field effects. Nature Neuroscience, 2(1), 79-87.

Rasin, E., Berger, I., Lan, N., Shefi, I., \& Katzir, R. (2021). Approaching explanatory adequacy in phonology using Minimum Description Length. Journal of Language Modelling, 9(1), 17-66.

Reuland, E. (2011). Anaphora and Language Design. Cambridge, MA: MIT Press.

Richards, M. (2011). Deriving the edge: what's in a phase? Syntax, 14, 74-95.

Richards, N. (2016). Contiguity Theory. Cambridge, MA: MIT Press.

Rissanen, J. (1989). Stochastic Complexity in Statistical Inquiry. River Edge, NJ: World Scientific Publishing Company.

Rizzi, L. (1990). Relativized Minimality. Cambridge, MA: MIT Press.

Rizzi, L. (2001). Relativized minimality effects. In M. Baltin \& C. Collins (Eds.), The Handbook of Contemporary Syntactic Theory (pp. 89-110). Oxford: Blackwell.

Roberts, I. (2019). Parameter Hierarchies and Universal Grammar. Oxford: Oxford University Press.

Romano, S., Sigman, M., \& Figueira, S. (2013). LT'2 $C^{2}$ : A language of thought with Turing-computable Kolmogorov complexity. Papers in Physics, 5, 050001.

Ruffini, G. (2017). An algorithmic information theory of consciousness. Neuroscience of Consciousness, 2017(1), nix019. 
NATURAL LANGUAGE SYNTAX COMPLIES WITH THE FREE-ENERGY PRINCIPLE

1620

1621

1622

1623

1624

1625

1626

1627

1628

1629

1630

1631

1632

1633

1634

1635

1636

1637

1638

1639

1640

1641

1642

1643

Samo, G. (2021). N-merge systems in adult and child grammars: a quantitative study on external arguments. Qulso, 7, 103-130.

Samuels, B.D. (2011). Phonological Architecture: A Biolinguistic Perspective. Oxford: Oxford University Press.

Schein, B. (1993). Plurals and Events. Cambridge, MA: MIT Press.

Schmidhuber, J. (2010). Formal theory of creativity, fun, and intrinsic motivation (1990-2010). IEEE Transactions on Autonomous Mental Development, 2(3), 230-247.

Schmidhuber, J. (2015). Deep learning in neural networks: an overview. Neural Networks, 61, 85-117.

Schütze, C.T.R. (1997). INFL in Child and Adult Language: Agreement, Case and Licensing. PhD dissertation, MIT.

Sengupta, B., \& Friston, K. (2018). How robust are deep neural networks? arXiv, 1804.11313.

Sengupta, B., \& Stemmler, M.N. (2014). Power consumption during neuronal computation. Proceedings of the IEEE, 102(5), 1-13.

Shieber, S. (1985). Evidence against the context-freeness of natural language. Linguistics and Philosophy, 8(3), 333-343.

Shumbayawonda, E., Tosun, P.D., Fernández, A., Hughes, M.P., \& Abásolo, D. (2018). Complexity changes in brain activity in healthy ageing: a permutation Lempel-Ziv complexity study of magnetoencephalograms. Entropy, 20, 506.

Smith, R., Parr, T., \& Friston, K.J. (2019). Simulating emotions: an active inference model of emotional state inference and emotion concept learning. Frontiers in Psychology 10: 2844.

Solomonoff, R.J. (1964). A formal theory of inductive inference. Part I. Information and Control, 7(1), 122.

Sprouse, J. (2011). A validation of Amazon Mechanical Turk for the collection of acceptability judgments in linguistic theory. Behavior Research Methods, 43, 155-167. 
NATURAL LANGUAGE SYNTAX COMPLIES WITH THE FREE-ENERGY PRINCIPLE

1644

1645

1646

1647

1648

1649

1650

1651

1652

1653

1654

1655

1656

1657

1658

1659

1660

1661

1662

1663

1664

1665

1666

1667

1668

Sprouse, J., \& Almeida, D. (2017). Setting the empirical record straight: acceptability judgments appear to be reliable, robust, and replicable. Behavioral and Brain Sciences, 40, e311.

Stabler, E.P. (1997). Derivational minimalism. In C. Retoré (Ed.), Logical Aspects of Computational Linguistics (pp. 68-95). LNCS 1328. New York: Springer-Verlag.

Starke, M. (2001). Move Dissolves into Merge. PhD thesis. University of Geneva.

Starke, M. (2004). On the inexistence of specifiers and the nature of heads. In A. Belletti (Ed.), The Cartography of Syntactic Structures, Vol. 3: Structures and Beyond (pp. 252-268). Oxford: Oxford University Press.

Steedman, M. (2000). The Syntactic Process. Cambridge, MA: MIT Press.

Sternefeld, W. (1997). Comparing reference sets. In C. Wilder, H-M. Gärtner, \& M. Bierwisch (Eds.), The Role of Economy Principles in Linguistic Theory (pp. 81-114). Berlin: Akademie Verlag.

Sundaresan, S. (2020). Distinct featural classes of anaphor in an enriched person system. In K. Hartmann, J. Mursell, \& P.W. Smith, P.W. (Eds.), Agree to Agree: Agreement in the Minimalist Programme (pp. 425-461). Open Generative Syntax series. Berlin: Language Science Press.

Szmrecsányi, B. (2004). On operationalizing syntactic complexity. In G. Purnelle, et al. (Eds.), Le poids des mots: Proceedings of the 7th International Conference on Textual Data Statistical Analysis, vol. 2 (pp. 1032-1039). Louvain-la-Neuve: Presses Universitaires de Louvain.

Tavano, A., Blohm, S., Knoop, C.A., Muralikrishnan, R., Scharinger, M., Wagner, V., Thiele, D., Ghitza, O., Ding, N., Menninghaus, W., \& Poeppel, D. (2021). Neural harmonics of syntactic structure. bioRxiv https://doi.org/10.1101/2020.04.08.031575.

Tervo, D.G.R., Tenenbaum, J.B., \& Gershman, S.J. (2016). Toward the neural implementation of structure learning. Current Opinion in Neurobiology, 37, 99-105.

Terzian, G., \& Corbalán, M.I. (2021). Simplicity of what? A case study from generative linguistics. Synthese, 198(10), 9427-9452.

Titov, E. (2020). Optionality of movement. Syntax, 23(4), 347-374. 
NATURAL LANGUAGE SYNTAX COMPLIES WITH THE FREE-ENERGY PRINCIPLE

1669

1670

1671

1672

1673

1674

1675

1676

1677

1678

1679

1680

1681

1682

1683

1684

1685

1686

1687

1688

1689

1690

1691

1692

1693 1694

Torr, J., Stanojević, M., Steedman, M., \& Cohen, S.B. (2019). Wide-coverage neural A* parsing for minimalist grammars. Processings of the 57th Annual Meeting of the Association for Computational Linguistics, Florence, Italy. 2486-2505.

Tribus, M. (1961). Thermodynamics and Thermostatics: An Introduction to Energy, Information and States of Matter, with Engineering Applications. New York: D. Van Nostrand Company Inc.

Trotzke, A., \& Zwart, J.-W. (2014). The complexity of narrow syntax: minimalism, representational economy, and simplest Merge. In F.J. Newmeyer, \& L.B. Preston (Eds.). Measuring Grammatical Complexity (pp. 128-147). Oxford: Oxford University Press.

Trudgill, P. (2011). Sociolinguistic Typology: Social Determinants of Linguistic Complexity. Oxford: Oxford University Press.

Turing, A. (1950). Computing machinery and intelligence. Mind, 49, 433-460.

Ungerleider, L.G., \& Haxby, J.V. (1994). 'What' and 'where' in the human brain. Current Opinion in Neurobiology, 4(2), 157-165.

Uriagereka, J. (2012). Spell-Out and the Minimalist Program. Oxford: Oxford University Press.

Urwin, S.G., Griffiths, B., \& Allen, J. (2017). Quantification of differences between nailfold capillaroscopy images with a scleroderma pattern and normal pattern using measures of geometric and algorithmic complexity. Physiological Measurement, 38(2), N32-N41.

Vaas, R. (2001). It binds, therefore I am! Review of Rodolfo Llinás's I of the Vortex. Journal of Consciousness Studies, 8(4), 85-88.

van Rooj, I., \& Baggio, G. (2021). Theory before the test: How to build high-versimilitude explanatory theories in psychological science. Perspectives on Psychological Science https://doi.org/10.1177/1745691620970604.

Vasil, J., Badcock, P.B., Constant, A., Friston, K., \& Ramstead, M.J.D. (2020). A world unto itself: human communication as active inference. Frontiers in Psychology, 11, 417.

Walkden, G., \& Breitbarth, A. (2019). Complexity as L2-difficulty: implications for syntactic change. Theoretical Linguistics, 45(3-4), 183-209. 
NATURAL LANGUAGE SYNTAX COMPLIES WITH THE FREE-ENERGY PRINCIPLE

1695

1696

1697

1698

1699

1700

1701

1702

1703

1704

1705

1706

1707

1708

1709

1710

1711

1712

1713

1714

1715

Wallace, C.S., \& Dowe, D.L. (1999). Minimum message length and Kolmogorov complexity. The Computer Journal, 42(4), 270-283.

Wexler, K. (2003). Lenneberg's dream: learning normal language development and specific language impairment. In J. Schaffer \& Y. Levy (Eds.), Language Competence across Populations: Towards a Definition of Specific Language Impairment (pp. 11-61). London: Lawrence Erlbaum.

Wilder, C., Gärtner, H.-M., \& Bierwisch, M. (Eds.). (1997). The Role of Economy Principles in Linguistic Theory. Berlin: Akademie Verlag.

Wilkinson, C., \& Murphy, E. (2016). Joint interventions in autism spectrum disorder: relating oscillopathies and syntactic deficits. UCL Working Papers in Linguistics, 28, 1-7.

Winn, J., \& Bishop, C.M. (2005). Variational message passing. Journal of Machine Learning Research, $6,661-694$.

Wipf, D.P., \& Rao, B.D. (2007). An empirical Bayesian strategy for solving the simultaneous sparse approximation problem. IEEE Transactions on Signal Processing, 55(7), 3704-3716.

Yang, C., Crain, S., Berwick, R.C., Chomsky, N., \& Bolhuis, J.J. (2017). The growth of language: universal grammar, experience, and principles of computation. Neuroscience and Biobehavioral Reviews, 81(B), 103-119.

Yngve, V. (1960). A model and a hypothesis for language structure. Proceedings of the American Philosophical Society, 104, 444-466.

Yun, J., Chen, Z., Hunter, T., Whitman, J., \& Hale, J. (2015). Uncertainty in processing relative clauses across East Asian languages. Journal of East Asian Linguistics, 24(2), 113-148.

Zhao, C. (2021). Chinese verb copying constructions: a labeling account. Lingua, 269, 103200. 